\title{
Higher-order chromatin organization defines PR and PAX2 binding to regulate endometrial cancer cell gene expression
}

\author{
Alejandro La Greca ${ }^{1}$ \\ Nicolás Bellora ${ }^{4 \ddagger}$ \\ Francois Le Dily $2 \ddagger$ \\ Rodrigo Jara ${ }^{1 \ddagger}$ \\ Javier Quilez Oliete ${ }^{2}$ \\ Gabriela Merino ${ }^{5}$ \\ Cristóbal Fresno ${ }^{5}$ \\ Inti Tarifa Rieschle ${ }^{1}$ \\ Griselda Vallejo ${ }^{1}$ \\ Guillermo Vicent ${ }^{2}$ \\ Elmer Fernández ${ }^{5}$ \\ Miguel Beato ${ }^{2,3}$ \\ Patricia Saragüeta ${ }^{1 *}$ \\ ${ }^{1}$ Biology and Experimental Medicine Institute, IBYME-CONICET, Buenos Aires, \\ Argentine. ${ }^{2}$ Centre for Genomic Regulation (CRG), Barcelona Institute for Science \\ and Technology, Barcelona, Spain. ${ }^{3}$ Universitat Pompeu Fabra (UPF), Barcelona, \\ Spain. ${ }^{4}$ Biodiversity and Environment Investigations Institute (INBIOMA), Bar- \\ iloche, Argentine. ${ }^{5}$ Bioscience Data Mining Group, Córdoba University, Córdoba, \\ Argentine. \\ ${ }^{\ddagger}$ Equal contributors *Corresponding author: patriciasaragueta2@gmail.com
}

\section{Running title}

$\mathrm{PR}$ and $\mathrm{ER} \alpha$ gene regulation in Ishikawa cells.

\section{Competing interest}

None declared.

\section{Funding}

This work was supported by the National Scientific and Technical Research Council (CONICET), Grant/Award Number: PIP 2015-682; Scientific and Technical Research Fund (FONCyT), Grant/Award Number: PICT 2015-3426. ERC-MINCYT international cooperation program. Doctoral Fellowship from CONICET, Argentina, awarded to ALG. PS is PI from CONICET. 


\title{
Higher-order chromatin organization defines PR and PAX2 binding to regulate endometrial cancer cell gene expression
}

\author{
Alejandro La Greca ${ }^{1}$, Nicolás Bellora ${ }^{4 \ddagger}$, Francois Le Dily ${ }^{2 \ddagger}$, Rodrigo \\ Jara $^{1 \ddagger}$, Javier Quilez Oliete ${ }^{2}$, Gabriela Merino ${ }^{5}$, Cristóbal Fresno ${ }^{5}$, Inti \\ Tarifa Rieschle $^{1}$, Griselda Vallejo ${ }^{1}$, Guillermo Vicent ${ }^{2}$, Elmer Fernández ${ }^{5}$, \\ Miguel Beato ${ }^{2,3}$, Patricia Saragüeta ${ }^{1 *}$ \\ ${ }^{1}$ Biology and Experimental Medicine Institute, IBYME-CONICET, Buenos Aires, \\ Argentine. ${ }^{2}$ Centre for Genomic Regulation (CRG), Barcelona Institute for Science and \\ Technology, Barcelona, Spain. ${ }^{3}$ Universitat Pompeu Fabra (UPF), Barcelona, Spain. \\ ${ }^{4}$ Biodiversity and Environment Investigations Institute (INBIOMA), Bariloche, \\ Argentine. ${ }^{5}$ Bioscience Data Mining Group, Córdoba University, Córdoba, Argentine. \\ ${ }_{\ddagger}^{\ddagger}$ Equal contributors ${ }^{*}$ Corresponding author: patriciasaragueta2@gmail.com
}

\begin{abstract}
Estrogen (E2) and Progesterone (Pg) via their specific receptors, ER and PR respectively, are major determinants in the development and progression of endometrial malignancies. We have studied how E2 and the synthetic progestin R5020 affect genomic function in Ishikawa endometrial cancer cells. Using ChIPseq in cells exposed to the corresponding hormones, we identified cell specific binding sites for ER (ERbs) and PR (PRbs), mostly binding to independent sites and both adjacent to PAXbs. Long-range interactions (HiC) showed enrichment of PRbs and PAXbs, which we call progestin control regions (PgCRs) inside TADs with differentially progestin-regulated genes. Effects of hormone treatments on gene expression were detected by RNAseq. PgCRs correlate with open chromatin independently of hormonal stimuli. In summary, endometrial response to progestins in differentiated endometrial tumor cells results in part from binding of PR to compartmentalized PgCRs in hormone-independent open chromatin, which include binding of partner transcription factors, in particular PAX2.
\end{abstract}

Keywords: steroid receptors, gene regulation, endometrial cancer, ChIPseq, HiC, ATACseq, progesterone receptor, estrogen receptor, PAX2 


\section{Introduction}

Progesterone $(\mathrm{Pg})$ is a key regulator in the female reproductive tract, including uterine and mammary gland development [1]. Endometrial and breast tissues exhibit significantly different responses to hormones, resulting in very distinctive morphologies and functions. During pregnancy, Pg prepares the uterine epithelium to receive the embryo and initiates the process of differentiation of stromal cells towards their decidual phenotype. In the mammary gland and in coordination with prolactin, Pg stimulates epithelial proliferation and differentiation of alveolar lobes in the mammary gland [2]. Unlike Pg, estradiol (E2) is the main proliferative signal in the uterine epithelium and exerts its function through activating estrogen receptor (ER) alpha and beta $(\operatorname{ER} \alpha$ and $\beta$, respectively) $[3,4]$.

The physiological role of $\mathrm{Pg}$ is mediated by the interaction and consequent activation of isoforms $\mathrm{A}(\mathrm{PRA})$ and $\mathrm{B}(\mathrm{PRB})$ of the progesterone receptor $(\mathrm{PR})$, which are transcribed from alternate promoters of the gene [5]. While PRA is more abundant in stromal endometrial cells, PRB is the most representative isoform in ephitelial cells of endometrium. Steroid hormones exert their transcriptional effects through binding of the steroid receptors (SR) to specific DNA sequences in the promoters or enhancers of target genes known as "hormone response elements" (HRE). Estradiol exposure triggers ER binding to estrogen response elements (ERE) regulating target genes such as $P G R$. Previous work showed E2-dependent upregulation of PR in many different target cells, species and pathological conditions [6, 7]. Exposure to progestins triggers binding of PR to PRE. Once bound to their HREs the hormone receptors interact with other transcription factors, co-regulators [8], such as the p160 family of co-activators of steroid receptors SRC-1-3, and chromatin remodelling enzymes. This evidence favors tissue specific roles of PR isoforms and their co-regulators orientated towards differential transactivation of target genes.

High levels of PRA and PRB have been described in endometrial hyperplasia [9] while low and high-grade endometrial cancers reveal reduced or absent expression of one or both isoforms in epithelia or stroma [10]. This PR decrease is often associated with shorter progression-free survival and overal survival rates $[11,9,12,13,14]$. The absence of PR gene expression may be attributed to hypermetilation of $\mathrm{CpG}$ islands within the promoter or first exon regions of the PR gene or to the presence of associated deacetylated histones. These modifications were reported for endometrial cancer cell lines as well as tumor samples and may be exclusive to PRB $[15,16,17]$. Treatment of such cells with DNA methyltranferase or histone deacetylase inhibitors can restore both $\mathrm{PRB}$ expression and its regulation of target genes such as FOXO1, p21 (CDKN1A), p27 (CDKN1B), and cyclin D1 (CCND1) [16, 18]. Down-regulation of PR by post-transcriptional mechanisms and through pos-translational modifications of PR may contribute to progesterone 
resistance in endometrial cancer but have not been extensively explored in the context of endometrial cancer. It is known that oncogenic activation of KRAS, PI3K or AKT and/or loss of functional tumor suppressors such as PTEN are common genetic alterations [19], toghether with $A R I D 1 A$ [20], all of them observed in endometrial cancer. Although there are numerous reports of hormonally regulated enhancers and super-enhancers in mammary cancer cells (see in dbsuperenahncer, http://bioinfo.au.tsinghua.edu.cn/dbsuper/) [21, 22], there is a void of information about their presence in endometrial cells.

To better understand the response to progestin in endometrial cancer cells, we have studied the genomic binding of ER and PR, the global gene expression changes and the state of chromatin by ATACseq as well as the genomic interactions by $\mathrm{HiC}$ in Ishikawa cells exposed to progestin or estrogen, and also in cells expossed to progestin after a period of estradiol pretreatment. Inside TADs with progestin regulated genes, we identified regions that we named progestin control regions (PgCRs) that correlate with the open chromatin compartment independently of hormonal stimuli and include binding sites for the partner transcription factor PAX2.

\section{Results}

\section{Ishikawa endometrial epithelial cells respond to $\mathrm{R} 5020$ through activation of $P R$, whose levels increase upon exposure to E2}

Endometrial epithelial cells respond to ovarian steroid hormones -progesterone (Pg) and estradiol (E2)-, E2 being the main proliferative stimulus and Pg its antagonist. After treating Ishikawa cells with E2 10nM for $48 \mathrm{~h}$ we observed an increment in number of cells compared to vehicle $(\mathrm{OH})(\mathrm{FC} 1.78 \pm 0.08 \mathrm{v}$. OH) that was suppressed by addition of R5020 10nM (FC 1.15 $\pm 0.08 \mathrm{v}$. OH) (Figure 1A). Treatment with R5020 10nM alone did not induce proliferation on Ishikawa cells $(\mathrm{FC} 0.77 \pm 0.08 \mathrm{v}$. OH) (Figure $1 \mathrm{~A})$. E2-induced cell proliferation was also abrogated by pre-incubation with estrogen receptor (ER) antagonist ICI182780 $1 \mu \mathrm{M}$ (ICI 10-6M) (FC $1.05 \pm 0.05$ v. OH) (Supplementary Fig. S1A), but not

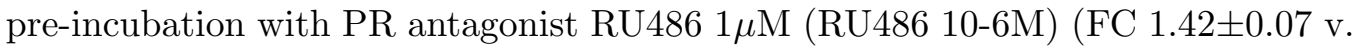
$\mathrm{OH})$ (Supplementary Fig. S1B), proving that ER but not PR was directly involved in the proliferative response to E2. Suppression of E2-induced cell proliferation by R5020 was inhibited by pre-incubation with RU486 (FC $1.50 \pm 0.06$ v. OH), indicating that R5020 effect was mediated by PR in Ishikawa cells (Supplementary Fig. S1B). The effects of E2 and R5020 on proliferation were corroborated by BrdU incorporation and cell cycle phase analysis $18 \mathrm{~h}$ after hormone exposure (Supplementary Fig. S1C and S1D). E2 increased the number of BrdU positive cells and percentage of cells in $\mathrm{S}$ phase compared to untreated control cells and to 
cell exposed to the vehicle $(\mathrm{OH})$, and these increments were inhibited by R5020. Treatment with the inhibitor of lysine acetyl transferase inhibitor Trichostatin A $250 \mathrm{nM}$ (TSA $250 \mathrm{nM}$ ) that blocks the cell cycle was used as negative control for BrdU incorporation and cell cycle progression (Supplementary Fig. S1C).

Ishikawa cells contain isoforms $\mathrm{A}$ and $\mathrm{B}$ of $\mathrm{PR}$ (PRA and PRB), both of which increased their steady state levels by treating cells with E2 10nM for 12h (Supplementary Fig. S1E and S1F). Pretreating cells with E2 for 12h (preE2) had little effect on the proliferative response to R5020 (Figure 1A), while E2 pre-treatment for $48 \mathrm{~h}$ significantly increased the proliferative effect of E2 exposure, compared to non-pretreated cells (FC $1.47 \pm 0.08 \mathrm{v}$. no preE2). The percentage of cells exhibiting nuclear localization of PR increased upon E2 pretreatment prior to R5020 exposure (T0). Upon exposure to R5020 for 60min the percentage of cells exhibiting nuclear PR was not affected by E2 pretreatment, though the intensity of the fluorescence signal increased in E2-pretreated cells (Figure 1B). Ishikawa cells express ER $\alpha$ but not ER $\beta$ (Supplementary Fig. S1G), suggesting that the proliferative effect of $\mathrm{E} 2$ was mediated by $\mathrm{ER} \alpha$. As was observed for hormone-bound PR, upon 60min of E2 exposure Ishikawa cells showed increased nuclear localization of $\operatorname{ER} \alpha$ (data not shown). R5020 increased nuclear ER $\alpha$ as well, suggesting a functional PR-ER crosstalk in response to hormonal stimuli (Supplementary Fig. $\mathrm{S} 1 \mathrm{H})$. Such interactions have already been proven in breast cancer T47D cells [23] and in UIII rat endometrial stromal cells [24], though in the latter PR remains strictly cytoplasmatic. Compared to T47D cells, PR protein levels in Ishikawa cells were significantly lower (Supplementary Fig. S2A and S2B). Copy number variation analysis revealed that Ishikawa cells carry neither chromosomal alterations nor multiple copies of the PGR (progesterone receptor) locus as was shown in T47D cells, possibly accounting for differences in PR content between both cell lines (Supplementary Fig. S2C; [25]).

To further support the functional activity of PR in Ishikawa cells, a reporter gene downstream of the MMTV promoter containing several Pg response elements (PREs) (Figure 1C) was transiently transfected and the cells were exposed to either R5020 10nM or vehicle $(\mathrm{OH})$ for 20 hours. A 2.8 fold increment in Luciferase activity was found in cells exposed to R5020 relative to $\mathrm{OH}$ controls, supporting the capacity of ligand-activated PR to activate PREs (Figure 1C). Binding of PR to cellular chromatin targets was confirmed by ChIP analysis of the PRE at the enhancer sequence located $50 \mathrm{~kb}$ upstream from the transcription start site (TSS) of the EGFR (epidermal growth factor receptor) gene (Figure 1D). Together, these observations demonstrate that $\mathrm{PR}$ is responsive to progestins and functional in Ishikawa cells under our culture conditions. 


\section{Endometrial PR and ER $\alpha$ binding}

To explore the genome-wide distribution of PR and ER $\alpha$ binding (PRbs and ERbs respectively) in Ishikawa cells, ChIPseq was performed in different conditions (Figure 2A and Supplementary Fig. S3). First, we analyzed untreated cells (T0) and cells exposed for 5, 30, and 60min to $10 \mathrm{nM}$ R5020 using an specific antibody to PR. Results showed robust PR binding after 30 min of R5020 treatment (R5020 $30 \mathrm{~min})$ with 1,446 sites, of which 322 sites $(22 \%)$ were present in untreated cells (PRbs at time zero, T0=331). After 60min of treatment with R5020 (R5020 $60 \mathrm{~min})$, the majority of sites identified at $30 \mathrm{~min}$ were still evident $(78 \%)$, with 336 sites gained and 307 sites lost (Figure 2A and Supplementary Fig. S3A). qPCR performed on six regions occupied by $\mathrm{PR}$ at 30 and 60min of R5020 exposure validated ChIPseq results (Supplementary Fig. S3B). Hormone-dependent increase in PR occupancy becomes steady at $60 \mathrm{~min}$ of treatment, coherence with qPCR results (Supplementary Fig. S3C). These results indicate that PR binding to chromatin is enhanced or stabilized by hormone and that maximum PR occupancy in Ishikawa cells is achieved at 30-60min of exposure to R5020.

Next we explored the recruitment of $\operatorname{ER} \alpha$ to chromatin in Ishikawa cells. ChIPseq with a specific ER $\alpha$ antibody was performed in untreated (T0) and in cells exposed to E2 (10nM) for 5, 60 and 360min. Poor ER $\alpha$ binding was detected at T0 (25 sites), of which $90 \%$ remained occupied by the ER $\alpha$ throughout all times of treatment with E2. Exposure to E2 resulted in the detection of 178 ER $\alpha$ binding sites (ERbs) at 5min, 1,591 at 60min and 1,973 at 360min (Figure 2A and Supplementary Fig. S3D), suggesting that ER $\alpha$ genomic action takes place and it is maintained mainly after hormone induction. The majority of ERbs in 5min-E2-treated samples was still present at 60 and 360min (data not shown) and nearly $85 \%$ of sites found at $60 \mathrm{~min}$ of $\mathrm{E} 2$ treatment were also identified at $360 \mathrm{~min}$ (Supplementary Fig. S3D). ER $\alpha$ binding at 0, 60 and 360min of E2 treatment was confirmed by qPCR on four of the sites identified (Supplementary Fig. S3E). ChIPseq results point to a clear and sustained E2-dependent enhancement or stabilization of ER $\alpha$ binding, which maintained between 60 and 360min of hormone exposure (Supplementary Fig. S3F).

De novo motif discovery confirmed that PR binding occurred mostly through PREs exhibiting the complete palindromic response elements (Supplementary Fig. S3G). ER binding sites were enriched in the half-palidromic ERE motifs (Supplementary Fig. S3H). Comparison with previous findings in T47D cells [26] enabled the clustering of both PRbs and ERabs into two clases (Supplementary Fig. S3G and $\mathrm{S} 3 \mathrm{H}$, respectively): sites specific for Ishikawa cells (group I; 595 PRbs, group III: 1101 ERbs) and sites present in both Ishikawa and T47D cell lines (group II: 896 PRbs; group IV: 490 ERbs)- revealed that while PR binds mostly through complete PREs, Ishikawa ERbs (group III) comprehend only half of the charac- 
teristic palindrome otherwise found in EREs.

\section{Estrogenic environment defines the landscape for $P R$ binding in endometrial cancer cells}

Sequencing experiments performed on T47D cells exposed to $10 \mathrm{nM}$ R5020 revealed over $25000 \mathrm{PRbs}[26,27]$, likely reflecting the high content of $\mathrm{PR}$ in these cells. However, a large proportion of these PRbs was considered not functionally relevant as indicated by the lack of nucleosome remodelling in response to hormone exposure [26]. More recent experiments in T47D exposed to subnanomolar R5020 have revealed that around 2,000 PRbs are sufficient to evoke a functional response (Zaurin et al, 2019, personal communication). Hence, the number of PRbs found in Ishikawa cells probably reflects the low concentration of PR but is compatible with a functional response to progestins. To test this possibility we increased the levels of PR in Ishikawa cells by expressing a recombinant FLAG-PR vector (Supplementary Fig. S4A). These cells, FPR Ishikawa (FPR), expressed levels of PR comparable to T47D cells (Supplementary Fig. S4B) and showed no impairment in hallmark phosphorylation of serine 294 in PR (Supplementary Fig. $\mathrm{S} 4 \mathrm{C}$ ), indicating that FPR cells were capable of responding to hormone. Upon hormone exposure FPR cells exhibited rapid binding of PR to the EGFR enhancer sequence (Supplementary Fig. S4D). ChIPseq experiments upon R5020 exposure showed twice the number of PRbs in FPR cells compared to the parental Ishikawa cells. The majority of PRbs identified in Ishikawa cells (>90\%) were also detected in FPR cells (Supplementary Fig. S4E), meaning that PR overexpression reflected mostly on an increase in number of binding sites.

Shifts in the synthesis and secretion of the ovarian steroids (estrogen and progesterone) during the menstrual cycle serve as the principal hormonal drivers for endometrial changes. Rising circulating estradiol during the mid-to-late follicular phase of the cycle promotes the proliferation of the functional endometrium, and higher E2 levels up-regulate $P G R$ gene expression [6, 7]. To explore the effect of E2 on PR behaviour we performed PR ChIPseq analyses in Ishikawa cells exposed for $12 \mathrm{~h}$ to E2 (10nM) (preE2) before exposure for 30 and $60 \mathrm{~min}$ to R5020 (10nM). Pretreatment with E2 significantly increased the intracellular levels of PR (numbers compared to FPR) as well as the number of R5020-dependent PRbs (prePRbs), which include most of PRbs already identified in non-pretreated Ishikawa cells (Figure 2A, Venn Diagram). Quantitative real-time PCR (qPCR) validations performed on 6 binding sites occupied by no-preE2 and preE2 PR confirmed positioning of the receptor in both conditions (Supplementary Fig. S4F). It also showed that E2 pretreatment augments both number of PRbs available for binding and occupancy of the receptor. Contrary to non-pretreated cells (both Ishikawa and FPR cells), PR binding continued to increase after $30 \mathrm{~min}$ in preE2 
cells and reached 5701 sites after 60 min of R020 exposure (Figure 2A and Supplementary Fig. S4G, S4H and S4I).

Upon hormone induction, sites engaged by PR in Ishikawa cells were also occupied in FPR and pretreated cells, denoting a strong similarity between them (Figure 2B). Using similarity between samples (Jaccard Statistic) as plotting distance clearly showed that FPR cells resembled parental Ishikawa cells more than they did pretreated cells. Although a small number of binding sites was shared between ER $\alpha$ and each PR group, PR binding in pretreated cells exhibited a higher degree of similarity to $\mathrm{ER} \alpha$ binding than FPRbs (Figure 2A Venn diagram and 2B). Moreover, subtracting PRbs from FPRbs (FPR_s) and prePRbs (prePR_s) heightens this difference (Figure 2B), with a much larger fraction of binding sites shared with $\mathrm{ER} \alpha$ in the case of prePRbs (Figure 2C). Among these sites, one located close to the promoter of TGFA gene (transforming growth factor alfa), identified as an ERbs, showed PR binding only in preE2 Ishikawa cells, but not in FPR (Figure 2D, left panel). ERE-containing ERbs, such as the ones found in the transcription termination site of $P G R$ gene and immediately upstream of $C C N D 2$ promoter, were occupied by R5020-bound PR in preE2 Ishikawa cells (Figure 2D, middle and right panels). These three genes were upregulated by E2 treatment in RNAseq experiments performed on Ishikawa cells (Supplementary Fig. S5). Taken together, this evidence suggests that, provided there is an estrogenic background, activated PR could regulate estrogen-dependent Ishikawa-specific transcriptome by binding sites already or formerly bound by $\operatorname{ER} \alpha$.

The distribution of PRbs and ERbs in non-pretreated Ishikwa cells, in FPR cells and cells pretreated with E2 (prePRbs) relative to TSS of regulated genes was consistent with previous reports in oher cell lines [26, 28], in that they were enriched in intronic and distal intergenic regions (Figure 3). Nearly $50 \%$ of binding sites localized to distal regions $(>50 \mathrm{~Kb})$ and approximately $30 \%$ to introns other than the first intron, indicating that regulation of gene expression by the steroid receptors $\mathrm{PR}$ and $\mathrm{ER} \alpha$ is not mediated through proximal promoters but by distal enhancer/silencer sequences.

As expected, from the sequences contained in $10 \mathrm{~kb}$ windows centered in peak summits of PRbs, FPRbs and prePRbs the PRE emerged as the most representative binding motif (Figure 4A), including sites uniquely found in FPR (group c: 633 ) or preE2 (group d: 3,162) cells. While comparison between ER $\alpha$ and PR ChIPseq results showed few similarities regarding identity of binding sites, with a set of 216 shared by both hormone receptors, pretreatment with E2 added nearly twice as many binding sites to the pool shared with ER $\alpha$ (Figure 2A, Venn Diagram). The most representative motif discovered in these sites -only shared by ER $\alpha$ and prePR- was a half ERE (Figure 4B, group h: 329) that was highly similar to the motif observed in sites uniquely found in Ishikawa ERbs (Supplementary 
Fig. S3H, group III). Sites shared by ER $\alpha$ and PR in all three conditions resulted in an unclear combination of PRE and ERE motifs (Figure 4B, group e-g). Degenerated motif logo in group g showed no association to any known motif, probably due to a corrupt analysis performed on insufficient data, and the partially degenerated motif logo in group e showed limited association to both PRE and ERE (PRE/ERE).

\section{$P A X 2$ binds chromatin in close proximity to $E R \alpha$ and PR binding sites in Ishikawa cells}

Evidence described so far partially explains cell type specific hormonedependent gene regulation, though it is not sufficient to understand the mechanisms underlying differential binding of hormone receptors to chromatin. Initially, we addressed this by contrasting the sequences of ERbs and PRbs from groups I-IV, i.e. hormone regulated Ishikawa specific, (Supplementary Fig. S3G and $\mathrm{S} 3 \mathrm{H}$ ) with an array of 1,395 known TF binding motifs (see Extended Methods in Supplemental Materials). Results revealed an enrichment ( $\mathrm{p}$-value $<1 \mathrm{e}^{-4}$ ) of multiple members of the PAX family -including variants 2, 5,6 and 9- in groups I and III, i.e. In Ishikawa specific PRbs and ERbs (Supplementary Fig. S6A and S6B, respectively), suggesting that members of the Pax family may be involved in $\mathrm{PR}$ and $\mathrm{ER} \alpha$ action in Ishikawa cells. Unbiased comparison of enrichment in TF binding motifs between Ishikawa and T47D PRbs showed similar results for PRbs, although the enrichment was less significant (Supplementary Fig. S6C). Moreover, while enrichment of PAX motifs was also observed around ERbs in Ishikawa cells (Supplementary Fig. S6D), this was not the case with T47D cells, in which examples like the well-known breast-related pioneer transcription factor FOXA1, were found instead (Supplementary Fig. S6E). Enrichment of NR3C1-4 (mineralocorticoid, glucocorticoid, progesterone and androgen receptors) and ESR1 motifs included into the 1,395 known motifs corroborated de novo discovery performed with MEME in both Ishikawa and T47D cells. Stronger enrichment of PAX motifs was observed in prePRbs compared to PRbs (Figure 4C), indicating that PR binding to regions potentially bound by PAX is favored after E2 pretreatment. Coherently, while equivalent fold enrichment values were detected when comparing prePRbs to ERbs (Figure 4D), comparison between prePRbs and FPRbs showed that increased PR levels alone were not sufficient for a greater association to PAX binding motifs (Supplementary Fig. S6F).

PAX association to PR and ER $\alpha$ action was also evaluated by immunofluorescence against PAX2. Nuclear localization of PAX2 was observed predominantly after $60 \mathrm{~min}$ of R5020 in pretreated and non-pretreated PR+ cells (Figure 5A), indicating that hormonal treatment promotes co-localization of PAX2 and PR in nuclei of Ishikawa cells. Similar results in PAX2 localization were obtained after 
treating Ishikawa cells with E2 (Figure 5B). The increase in nuclear PAX2 signal is not due to changes in protein levels, which were not affected by treatment with either R5020 or E2 (Supplementary Fig. S6G). In accordance to motif analysis results, PAX2 was not detected in nuclei of T47D cells after hormonal treatments (Supplementary Fig. S6H).

To extend these findings, we performed PAX2 ChIPseq experiments on untreated cells and in cells exposed for 60min to either R5020 or E2. The results confirmed PAX2 binding to chromatin following hormonal treatment (Supplementary Fig. S6I). Even though identified PAXbs were few (T0: 43, R60: 201 and E60: 208), most of PAX2 binding occurred after R5020 and E2 treatments. Moreover, PAX2 binding was not stochastically distributed in the genome of Ishikawa cells but rather partially associated to ERbs and PRbs. This association was stronger for PR binding in cells pre-teated with E2 than in non-pretreated cells or in cells overexpressing recombinant PR (Figure 5C). Similar results were obsereved for ERbs in resonse to E2 (Figure 5C and 5D), indicating that PAX2, and possibly other members of the PAX family may co-operate with PR and ER $\alpha$ for binding to chromatin in Ishikawa cells but not in T47D cells, in which neither enrichment for PAX binding motif nor nuclear localization of PAX2 was detected.

\section{Under estrogenic conditions PR and PAX2 conform endometrial regulatory domains in open chromatin compartments}

Nuclear architecture is a major determinant of hormonal gene regulatory patterns [25]. Therefore, we used in nucleo Hi-C technology to study the folding of chromatin across the genome of Ishikawa cells by generating genome-wide contact datasets of cells untreated (T0) or pretreated with E2 for 12h, and exposed for 60 minutes to R5020 or E2. A comparison of contact matrices at $20 \mathrm{~kb}$ resolution of untreated Ishikawa cells to T47D cells confirmed the high degree of conservation in topologically associating domains (TADs) borders (Supplementary Fig. S7A). TADs are grouped into two chromatin compartments $\mathrm{A}$ and $\mathrm{B}$, which represent the active open chromatin (A) and the closed inactive chromatin (B), respectively. Analysis of such compartments showed a cell type-specific patterning (Supplementary Fig. S7B), in which Ishikawa samples from two independent experiments were more closely related to each other than any of them to a T47D sample (Supplementary Fig. S7C and S7D). However, A/B profile distribution in Ishikawa cells was independent of hormonal treatments (Supplementary Fig. S7B and S7E), meaning that chromatin was in a primed state that conditioned hormone-dependent regulation of gene expression. Detailed analysis revealed that $7 \%$ of A domains in Ishikawa cells were B in T47D cells, and $12 \%$ of B domains in Ishikawa cells were A in T47D cells (Supplementary Fig. S7F). A total of 861 genes encompassed in the A compartment in Ishikawa cells belong in the B compartment in T47D cells, and 
1,438 genes in B compartaments in Ishikawa cells belong in A in T47D cells (12\%), suggesting that distribution of A and B compartments could in part explain cell type specific gene expression profiles.

To evaluate whether chromatin states is related to gene expression through differential binding of hormone receptors to DNA, we intersected PR and ER $\alpha$ ChIPseq results with the A/B compartment coordinates. Both transcription factors, $\mathrm{PR}$ and $\mathrm{ER} \alpha$, bound A compartments more frequently than $\mathrm{B}$, meaning that open genomic regions in Ishikawa showed preferential binding of the hormone receptors (Supplementary Fig. S7G). Neither pre-treatment with E2 nor expression of recombinant PR modified the preferential binding of the PR to the A compartments.

As mentioned above PAX2 binding occurs mostly in close proximity to PR and ER $\alpha$ binding sites. In fact, distances between PAXbs and PRbs were remarkably shorter in E2 pretreated cells than in any other condition (Figure 6A). This raised the question of whether recruitment of PR together with PAX2 to open chromatin compartments facilitates regulation of gene expression. To study this notion, we defined putative endometrial regulatory domains that we will name "Progestin Control Regions" (PgCR) with the capacity to potentially regulate nearby genes. The restrictions for being a regulatory domain, which consisted in containing at least two PRbs separated by a maximun distance of $25 \mathrm{~kb}$ and a PAXbs (represented in scheme above Figure 6B), were met mostly under E2 pretreated conditions (Figure 6B). This outcome was due to the strong association between prePRbs and PAXbs, though it may have been aided by the increased PR protein levels. However, the sole increment in PR protein levels was not enough to force an association to PAXbs, given that FPR cells did not show similar results (Figure $6 \mathrm{~B})$.

Considering that TAD borders may act as regulatory barriers, we removed from further analysis any region that satisfied the rules for being a $\mathrm{PgCR}$ while being localized across a barrier as well. In agreement with this restriction, the sizes of PgCR -with an average of $25 \mathrm{~kb}$ - were smaller than TADs -with an average of 1000kb- (Figure 6C). In addition, the majority of the 171 identified PgCRs were not located near the TAD borders, but in the TAD center (Figure 6D), where most non-housekeeping genes are found [29]. Moreover, PgCRs seem to be located in A compartments in the vecinity of hormone-regulated genes like PGR and ALPP (Figure 6E). Expression of these genes was analyzed by qPCR of total RNA samples of Ishikawa cells exposed to hormone for 12h-hormone and showed that ALPP is induced by both hormones and PGR is only induced by E2 (Supplementary Fig. S7H).

As was mentioned before, the $\mathrm{HiC}$ matrices were used to determine the spatial segregation of chromatin in both open and closed chromatin compartments (A/B), 
and the A:B ratio was independent of hormone treatment. Consistent with these results, ATACseq signal on PgCRs remained unchanged upon hormone exposure, but it decreased after shuffling the coordinates for PgCRs, indicating that chromatin was readily and non-randomly accesible to TFs in these locations (Figure $6 \mathrm{~F}$, top panels). Although ATACseq peaks were also detected on promoters of hormone-regulated genes, the signal did not differ after hormone exposure (Figure $6 \mathrm{~F}$, bottom panels), implying that treatments were not responsible for opening the chromatin in these regions. In addition, more than half of hormone-regulated genes were concentrated under 10 megabases away from PgCRs $(52 \%$ in R5020 exposed cells and 51\% in E2 exposed cells), including the genes with higher fold change values (Figure 6G).

These results suggest that specific binding of $\mathrm{PR}$ and $\mathrm{ER} \alpha$ to chromatin occurs in compartment that are present in a permissive (open) or restrictive (closed) status depending on the cell line, and are not modified by short term hormone exposure. Summing up, PR and ER bind mostly to non-common sites that exhibit the corresponding consensus sequences, and are adjacent to PAX2 binding. Therefore, the endometrial specific hormone response results in part from specific chromatin compartments, unique receptor binding sites and selective TFs binding partners to regulate gene expression.

\section{Discussion}

There seems to be consensus that the different manners in which TFs assemble to recognize binding sites and control transcription is "paramount to understanding their physiological roles, decoding specific functional properties of genomes, and mapping how highly specific expression programs are orchestrated in complex organisms" [30]. There is evidence that the same TF can regulate different genes in different cell types (e.g. ESR1 in breast and endometrial cell lines) [31], but the mechanisms through which hormone receptors regulate endometrial specific networks had not been previously deciphered. Here we describe ER and PR binding to endometrial Ishikawa cell genome and their specific chromatin context.

The expression of $T G F \alpha$ mRNA and secretion of TGF $\alpha$ in Ishikawa cells were induced by estradiol but not those in hormone independent HEC-50 cells [32].Our results on hormone-regulated gene expression, which include estradiol regulated TGF $\alpha$ mRNA, and the presence of ER and PR indicate that Ishikawa cells that we are using behave as was established when the cell line developed and can be considered a good model of Type I epithelial endometrial cancer [33].

Regarding the role of $\mathrm{PR}$ in endometrial function, it was reported that the absence of PR in PGR Knockout (PRKO) mice results in unopposed estrogeninduced endometrial hyperplasia [1]. PRB is considered a strong transcriptional 
activator while PRA functions as a transcriptional inhibitor of PRB activity. Selective ablation of PRA in mice results in a PRB dependent gain of function, with enhanced estradiol-induced endometrial proliferation [34, 35]. Ishikawa cells express more PRB than PRA (Supplementary Fig. S2A), coherent with PRB dominance in glandular epithelial cells [36].

To explore the mechanism underlying the endometrial specific response to ovarian steroids hormones, we studied the genomic binding of ER and PR by ChIPseq in hormone untreated Ishikawa cells and in cells exposed to hormone for different time periods. We discovered that the majority $(67 \%)$ of PRbs after estradiol pretreatment were new sites not present in untreated cells and different as well from ERbs occupied after estradiol treatment. Just 639 bs (11\% of all PRbs) were the same for both PR and ER. This indicates that contrary to what was described in breast cancer cells [37, 38], in endometrial cells PR binding has little effect on ER binding. In Ishikawa cells the binding of ER and PR occurs mainly at ERE and PRE sequences, respectively, in regions that are also enriched in PAX response elements. Ishikawa cells are rich in PAX TF and the PAX ChIPseq shows a similar overlapping with ERbs and PRbs.

In this study we analyzed the chromatin topology of Ishikawa cells using $\mathrm{HiC}$ and found PRbs and ERbs enriched in Topologically Associating Domains (TADs) containing hormone regulated genes. We found that these TADs were mainly part of the open (A) chromosome compartment, even in cells not exposed to hormone. This was confirmed using ATACseq, suggesting that mechanisms or TFs independent of ligand activated hormone receptors occupied the hormone responsive TADs and maintain them as open chromatin before exposure to hormone.

In that respect it is interesting that we find an enrichement of PAX2bs near PRbs in TADs containing progesterone regulated genes. Loss of PAX2 expression has been implicated in the development of endometrial intraepithelial neoplasia (EIN) [39] and PAX2 is potentialy useful in the diagnose of difficult EIN cases (e.g. where there is no "normal" tissue in a sample to act as an internal control when assessing nuclear morphology) [40]. Our results connect PR response elements with PAX2, which is consistent with progestin regulation preserved in differentiated cancer cells; this could be lost as tumor cells become undifferentiated, also losing hormone receptors.

We have found that PR-PAX-PRbs containing regulatory domains or PgCRs could reflect PR shadow enhancers [41] in endometrial cells. The redundancy of PRbs associated to endometrial specific gene expression may reinforce a genetic mechanism to ensure progestin regulation in tissue under hormonal influence. The only super enhancer described and deregulated so far in endometrial carcinomas is Myc super enhancer [42]; therefore, we named a useful and novel subset of 171 endometrial regulatory domains strategical in this cancer cell line. This could 
guide treatment to recover progestin regulation over estrogen proliferative effects in endometrial malignancy.

We have found that PR-PAX-PR binding sites containing regulatory domains or PgCRs could reflect PR shadow enhancers [41] in endometrial cells. The redundancy of PRbs associated to endometrial specific gene expression may reinforce a genetic mechanism to ensure progestin regulation in tissue under hormonal influence, e.g. in periods in which there is low or no cirulating hormone. The only super enhancer described and deregulated so far in endometrial carcinomas is Myc super enhancer [42]; therefore, we named a useful and novel subset of 171 endometrial regulatory domains strategical in this cancer cell line. This could guide treatment to recover progestin regulation over estrogen proliferative effects in endometrial malignancy.

Previous results in mammary cancer cells have shown Hormone Control Regions, which include ERbs and PRbs interacting with promoters of hormone regulated genes in T47D cells [29]. Analysis of interaction between PgCR and different ER enriched binding regions in endometrial cells remains to be performed. Our study focused primarily on PR sites originated under estrogenic conditions together with PAX2 encompassing progestin control regions, which might regulate endometrial specific gene expression.

\section{Methods}

\section{Cell culture and hormonal treatments}

Endometrial adenocarcinoma Ishikawa cells and FPR Ishikawa cells were cultured in phenol red DMEM/F12 medium (GIBCO, Thermo Fisher Scientific) supplemented with 10\% FCS (GreinerBioOne) and gentamycin (Thermo Fisher Scientific) at $37^{\circ} \mathrm{C}$ and $5 \%$ carbon dioxide to maintain cell line stock. Before each experiment, cells were plated in phenol red-free DMEM/F12 medium supplemented with $5 \%$ dextran-coated charcoal-treated (DCC)-FCS and gentamycin for $48 \mathrm{~h}$. Then, the medium was replaced by serum-free DMEM/F12 and kept in it for $18 \mathrm{~h}$ (overnight). Treatments were performed with R5020 and E2 to a final concentration of $10 \mathrm{nM}$ and ethanol (vehicle) for the times indicated for each experiment. When indicated, pretreatment with E2 consisted of a single administration of E2 to a final concentration of $10 \mathrm{nM} 12 \mathrm{~h}$ before hormonal treatments. T47D cells were cultured in RPMI 1640 medium as previously described [27].

\section{Transfection with flag-tagged PR (FPR Ishikawa cells)}

Plasmid p3xFLAG-CMV-14 carrying the complete sequence for progesterone receptor gene (HindIII924 - 938EcoRI) was introduced in Ishikawa cells using Lipofectamine 2000 (Thermo Fisher Scientific) following manufacturer recommendations. After $24 \mathrm{~h}$ of transfection, cells were exposed to $0.6 \mathrm{mg} / \mathrm{ml}$ G418 for selection. 
Then on, every two passages, FRP cells were exposed to a reduced concentration of G418 $(0.4 \mathrm{mg} / \mathrm{ml})$, except during hormonal treatments.

\section{Proliferation assay}

Ishikawa cells were seeded at $5 \times 10^{4}$ cells/plate density in $35 \mathrm{~mm}$ dish plates. After $48 \mathrm{~h}$ in $5 \%$ DCC-FCS, the medium was replaced for $1 \%$ DCC-FCS for $18 \mathrm{~h}$. Treatments were performed for $48 \mathrm{~h}$ and cells were then collected using trypsin (0.25\%). Antagonists for ER and PR, ICI182780 and RU486 $1 \mu \mathrm{M}$ respectively, were added for $60 \mathrm{~min}$ and removed before hormonal treatments. The number of live cells was determined using trypan blue $(0.1 \%)$ in Neubauer chamber, repeating the procedure sixteen times for each sample and performing three independent experiments.

\section{BrdU incorporation assay and cell cycle analysis}

Ishikawa cells were seeded and prepared for hormonal treatments as described for Proliferation assay. Treatments were carried out for $15 \mathrm{~h}$, the last two hours of which includes incubation with BrdU. Cells were treated with cell cycle inhibitor TSA A 250nM as negative control of BrdU incorporation. After collecting cells in trypsin and washing them with PBS, ethanol $70 \%$ was added to fix and permeabilize them. DNA denaturation was achieved with $0.5 \%$ BSA and $2 \mathrm{M} \mathrm{HCl}$ after which cells were incubated in 1:2000 solution of anti-BrdU (BD Pharmingen) for $1 \mathrm{~h}$ at RT. FITC secondary antibody (Dako) was incubated for $1 \mathrm{~h}$ in obscurity at RT followed by propidium iodide for $5 \mathrm{~min}$. BrdU incorporation and cell cycle phases were evaluated by flow cytometry (BD FACS Canto II) in three replicates.

\section{Western blot}

Cell extracts were collected at the times indicated by the experiment with $1 \%$ SDS, 25mM Tris- $\mathrm{HCl} \mathrm{pH} 7.8,1 \mathrm{mM}$ EDTA, 1mM EGTA and protease and phosphatase inhibitors. Total protein extracts were loaded in 8\% SDS-PAGE and incubated with the following antibodies: PR (H190, Santa Cruz Bio.), ER $\alpha$ (HC20, Santa Cruz Bio.) and $\alpha$-tubulin (Sigma Aldrich). Quantification of gel images was performed with ImageJ software and expressed as abundance in relative units to $\alpha$-tubulin.

\section{Immunofluorescence}

Cells were seeded onto coverslips in six-well plates in a density of $10^{3}$ cells $/ 150 \mu \mathrm{l}$ using the protocol described in Cell culture and hormonal treatments and either pretreated or not with E2 10nM during the last $12 \mathrm{~h}$ of serum-free culture. After hormonal treatments cells were washed with ice cold PBS followed by fixation and permeabilization by incubation in $70 \%$ ethanol for $12 \mathrm{~h}$ at $-20^{\circ} \mathrm{C}$. After rinsing 
three times for $5 \mathrm{~min}$ in $0.1 \%$ Tween-PBS, the coverslips were incubated for $2 \mathrm{~h}$ with $10 \%$ BSA in $0.1 \%$ Tween-PBS to reduce nonspecific staining. To detect PR (H190 Santa Cruz Bio.), phosphoserine 294 PR (S294 Cell Signaling), ER $\alpha$ (HC-20 Santa Cruz Bio.) and PAX2 (Biolegends) cells were incubated with corresponding antibodies diluted in $10 \%$ BSA $0.1 \%$ Tween-PBS at $4^{\circ} \mathrm{C}$ overnight. After several washes in Tween-PBS, coverslips were exposed to secondary antibodies Alexa 488 and Alexa 555 (Thermo Fisher Scientific, Thermo Fisher Scientific) diluted 1:1000 in $10 \%$ BSA $0.1 \%$ Tween-PBS for $1 \mathrm{~h}$ at room temperature using DAPI to reveal nuclei. Coverslips were mounted on slides with Mowiol mounting medium (Sigma Aldrich) and analyzed in TIRF Olympus DSU IX83 (Olympus Life Sciences Solutions). Quantification of nuclear fluorescence was done with ImageJ software after generating a binary mask in dapi images.

\section{$q R T P C R$}

After $12 \mathrm{~h}$ of treatment with R5020 and E2, cell extracts were collected in denaturing solution (4M Guanidine thiocyanate, $25 \mathrm{mM}$ Sodium citrate $\mathrm{pH} 7,0.1 \mathrm{M}$ 2-Mercaptoethanol, 0.5\% Sarkosyl) and total RNA was prepared following phenolchloroform protocol [43]. Integrity-checked RNA was used to synthesize cDNA with oligodT (Biodynamics) and MMLV reverse transcriptase (Thermo Fisher Scientific). Quantification of candidate gene products was assessed by real-time PCR. Expression values were corrected by GAPDH and expressed as mRNA levels over time zero (T0). Primer sequences are available on request.

\section{Luciferase reporter assay}

Ishikawa cells were seeded and prepared for hormonal treatments as described for Proliferation assay without addition of gentamycin. Cells were co-transfected with MMTV LTR-Firefly Luciferase (pAGMMTVLu, gift from Laboratory of Patricia Elizalde) and CMV-Renilla luciferase (pRL-CMV, Promega) plasmids using lipofectamine plus 2000 (Thermo Fisher Scientific). After 5h, media were renewed with the addition of antibiotics and $12 \mathrm{~h}$ later cells were treated with vehicle (ethanol) and R5020 for 20h. Firefly and Renilla activities (arbitrary units) were determined with Dual-Luciferase Reporter assay system (Promega) and expressed as Firefly units relative to internal control Renilla for each sample (Firefly x10 $/$ Renilla).

\section{RNAseq}

Total RNA was collected from untreated (T0) and 12h R5020- and E2-treated Ishikawa cells using RNeasy Plus Mini Kit (QIAGEN) and subjected to highthroughput sequencing in Illumina HiSeq 2000. Poly-A-enriched RNA was used to 
prepare libraries with TruSeq RNA Sample Preparation kit v2 y v4 (ref. RS-1222001/2, Illumina) according to instructions from manufacturer followed by singleend sequencing. Good quality $50 \mathrm{bp}$ reads were aligned to the reference human genome (hg19, UCSC) using Tophat software [44] keeping those that mapped uniquely to the reference with up to two mismatches and transcript assembly, abundance quantification and differential expression analyses were performed with the Cufflinks tool [45]. Genes under 200bp in length or with FPKM values below 0,1 were excluded from downstream analyses. Genes were classified into induced, repressed or non-regulated depending on $\log 2 \mathrm{FC}$ value relative to untreated cells (T0). Threshold value was arbitrarily set at $\log 2 \mathrm{FC}= \pm 0.8$ and $\mathrm{q}<0.05$ (FDR). Enriched terms were determined through RDAVIDWebservice [46] and WebGestalt (IBL, Zhang Lab.) under standard parameter settings for each tool.

\section{Chromatin immunoprecipitation (ChIP)}

ChIP experiments were performed as described in [47] and [48]. Antibodies used for immunoprecipitation were PR (H190, Santa Cruz Bio.), ER $\alpha$ (HC20X and H184X, Santa Cruz Bio.) and normal rabbit IgG (sc-2027, Santa Cruz Bio.). Enrichment to DNA was expressed as percentage of input (nonimmunoprecipitated chromatin) relative to untreated Ishikawa cells (T0) using the comparative $\mathrm{Ct}$ method. Ct values were acquired with BioRad CFX Manager software.

\section{ChIPseq}

After minor modifications to the ChIP protocol described in [48], purified ChIP-DNA was submitted to deep sequencing using Illumina HiSeq-2000. Libraries were prepared by the Genomics unit of the CRG Core Facility (Centre for Genomic Regulation, Barcelona, Spain) with NEBNext ChIPseq Library Prep Reagent Set (ref. E6200S, Illumina) and 50bp sequencing reads were trimmed to remove Illumina adapters and low-quality ends using Trimmomatic [49] version 0.33 in single-end mode. Good quality reads were aligned to the reference human genome (hg19, UCSC) with BWA [50] v0.7.12 (BWA_MEM algorithm with default parameters) keeping alignments that mapped uniquely to the genome sequence (Samtools version 1.2, [50]). Overlapping reads were clustered and significant signal enrichments (peaks) were identified by MACS2 v2.1.0 [51] using input as background signal. FDR value during initial peak calling steps was set to 0.05 (q), though downstream analyses included only those with $\mathrm{q}<10^{-5}$. When necessary peak files were converted to hg38 coordinates using the batch conversion tool from UCSC. 


\section{Heatmaps, Scatterplots and Motif analysis}

Overlap of ChIPseq peak regions defined by upstream peak calling procedures (MACS2) were determined using intersectBed program from the bedTools suite [52]. An overlap of at least one bp was considered positive. De novo motif discovery (MEME software) performed on sequences contained in 10kb windows centered in peak summits. Graphs, correlation tests, non-linear regression and statistical analyses in general were performed for common peaks between ChIPseq samples using R (R Development Core Team). Heatmaps were plotted using the summit of the peaks as a reference central position. Reference positions were taken from common and exclusive peaks within experiments and were sorted by height of the peak. Genome aligned reads occurring between -5000 and +5000 bp from reference sites were mapped using count_occurences program [53] and the number of reads per bins of 200bp was used for the color intensity of heatmap cells with R. For Motif discovery, genomic regions of top 500 peaks ranked by their height were extracted from each set and regions that overlap with repeats, low complexity regions or transposable elements (extracted from the UCSC genome browser, hg19 human assembly), were removed from the analysis. Motif discovery was performed using MEME program suite executed with the following parameters: -maxsize 250000 -revcomp -dna -nmotifs 3 -mod oops [54]. Motif enrichments were evaluated with the procedure and statistics described in [55]. Additionally, the analysis utilized a 5mers collection of 1,395 human position frequency matrices modelling transcription factors binding sites [56], which were scanned $\left(\mathrm{p}\right.$-value $\left.<1 \mathrm{e}^{-4}\right)$ and their enrichment evaluated in regions of 200bp centered in the summits of whole peaks sets. To uncover motif profiles, discovered and library motifs were wholegenome scanned ( $\mathrm{p}$-value $\left.<1 \mathrm{e}^{-4}\right)$. Their occurrences around the sets of summits were obtained with count_occurrences $( \pm 2000 \mathrm{bp}$, bin size $=200 \mathrm{bp})$ and the profiles showing the proportion of regions per bin having at least one match were plotted using R.

\section{ATACseq}

ATACseq was performed as previously described [57]. Briefly, 50,000 cells were lyzed with $50 \mu \mathrm{l}$ cold lysis buffer (Tris-Cl pH $7.410 \mathrm{mM} ; \mathrm{NaCl}$ $10 \mathrm{mM} ; \quad \mathrm{MgCl} 2 \quad 3 \mathrm{mM} ; \quad \mathrm{NP}-40 \quad 0.1 \% \mathrm{v} / \mathrm{v}$ ) and centrifuged at $500 \mathrm{xg}$ for $10 \mathrm{~min}$ at $4^{\circ} \mathrm{C}$. Nuclei were resuspended in TD Buffer with $1.5 \mu \mathrm{l}$ Tn5 Transposase (Nextera, Illumina) and incubated 15 minutes at $37^{\circ} \mathrm{C}$. DNA was isolated using Qiagen MinElute column and submitted to $10 \mathrm{cy}$ cles of PCR amplification using NEBNext High-Fidelity 2X PCR Master Mix (Univ. primer: AATGATACGGCGACCACCGAGATCTACACTCGTCGGCAGCGTCAGATGTG ; Indexed primers: CAAGCAGAAGACGGCATACGA- 
GATNNNNNNNNGTCTCGTGGGCTCGGAGATGT). Library were size selected using AMPure XP beads and sequenced on a NextSeq 500 instrument (2x75 nt).

$\mathrm{HiC}$

High-throughput conformation capture assays were performed as previously described [58]. Preparation, amplification and purification of $\mathrm{HiC}$ libraries were performed following Illumina specifications for pair-end sequencing in HiSeq200 platform (75bp reads) using libraries with no less than $10 \mathrm{ng} / \mu \mathrm{l}$. Data analyses and interpretation were done in collaboration with Francois Le Dily and Javier Quilez Oliete (Centre for Genomic Regulation, Barcelona, Spain).

\section{References}

[1] J. P. Lydon, F. J. DeMayo, C. R. Funk, S. K. Mani, A. R. Hughes, C. A. Montgomery, Jr, G. Shyamala, O. M. Conneely, B. W. O’Malley, Mice lacking progesterone receptor exhibit pleiotropic reproductive abnormalities, Genes Dev 9 (18) (1995) 2266-78.

[2] B. Mulac-Jericevic, O. M. Conneely, Reproductive tissue selective actions of progesterone receptors, Reproduction 128 (2) (2004) 139-46. doi:10.1530/rep.1.00189.

[3] I. Ishiwata, K. Kiguchi, C. Ishiwata, M. Soma, T. Nakaguchi, I. Ono, T. Tachibana, H. Hashimoto, H. Ishikawa, S. Nozawa, Histogenesis of hollow cell ball structure of ovarian and endometrial adenocarcinoma cells in vivo and in vitro, Hum Cell 10 (3) (1997) 209-16.

[4] U. A. Kayisli, J. Luk, O. Guzeloglu-Kayisli, Y. Seval, R. Demir, A. Arici, Regulation of angiogenic activity of human endometrial endothelial cells in culture by ovarian steroids, J Clin Endocrinol Metab 89 (11) (2004) 5794-802. doi:10.1210/jc.2003-030820.

[5] A. R. Hovland, R. L. Powell, G. S. Takimoto, L. Tung, K. B. Horwitz, An n-terminal inhibitory function, if, suppresses transcription by the a-isoform but not the b-isoform of human progesterone receptors, J Biol Chem 273 (10) (1998) 5455-60. doi:10.1074/jbc.273.10.5455.

[6] J. D. Graham, S. D. Roman, E. McGowan, R. L. Sutherland, C. L. Clarke, Preferential stimulation of human progesterone receptor b expression by estrogen in t-47d human breast cancer cells, J Biol Chem 270 (51) (1995) 30693700. 
[7] W. L. Kraus, B. S. Katzenellenbogen, Regulation of progesterone receptor gene expression and growth in the rat uterus: modulation of estrogen actions by progesterone and sex steroid hormone antagonists, Endocrinology 132 (6) (1993) 2371-9. doi:10.1210/endo.132.6.8504742.

[8] M. Beato, P. Herrlich, G. Schütz, Steroid hormone receptors: many actors in search of a plot, Cell 83 (6) (1995) 851-7.

[9] T. Miyamoto, J. Watanabe, H. Hata, T. Jobo, M. Kawaguchi, M. Hattori, M. Saito, H. Kuramoto, Significance of progesterone receptor-a and -b expressions in endometrial adenocarcinoma, J Steroid Biochem Mol Biol 92 (3) (2004) 111-8. doi:10.1016/j.jsbmb.2004.07.007.

[10] R. Shao, Progesterone receptor isoforms a and b: new insights into the mechanism of progesterone resistance for the treatment of endometrial carcinoma, Ecancermedicalscience 7 (2013) 381. doi:10.3332/ecancer.2013.381.

[11] K. K. Leslie, N. S. Kumar, J. Richer, G. Owen, G. Takimoto, K. B. Horwitz, C. Lange, Differential expression of the $\mathrm{a}$ and $\mathrm{b}$ isoforms of progesterone receptor in human endometrial cancer cells. only progesterone receptor $b$ is induced by estrogen and associated with strong transcriptional activation, Ann N Y Acad Sci 828 (1997) 17-26. doi:10.1111/j.1749-6632.1997.tb48520.x.

[12] H. Sakaguchi, J. Fujimoto, B. L. Hong, Y. Nakagawa, T. Tamaya, Drastic decrease of progesterone receptor form b but not a mrna reflects poor patient prognosis in endometrial cancers, Gynecol Oncol 93 (2) (2004) 394-9. doi:10.1016/j.ygyno.2004.01.042.

[13] V. Jongen, J. Briët, R. de Jong, K. ten Hoor, M. Boezen, A. van der Zee, H. Nijman, H. Hollema, Expression of estrogen receptor-alpha and beta and progesterone receptor-a and -b in a large cohort of patients with endometrioid endometrial cancer, Gynecol Oncol 112 (3) (2009) 537-42. doi:10.1016/j.ygyno.2008.10.032.

[14] H. Kreizman-Shefer, J. Pricop, S. Goldman, I. Elmalah, E. Shalev, Distribution of estrogen and progesterone receptors isoforms in endometrial cancer, Diagn Pathol 9 (2014) 77. doi:10.1186/1746-1596-9-77.

[15] M. Sasaki, A. Dharia, B. R. Oh, Y. Tanaka, S. Fujimoto, R. Dahiya, Progesterone receptor $\mathrm{b}$ gene inactivation and cpg hypermethylation in human uterine endometrial cancer, Cancer Res 61 (1) (2001) 97-102. 
[16] Y. Xiong, S. C. Dowdy, J. Gonzalez Bosquet, Y. Zhao, N. L. Eberhardt, K. C. Podratz, S.-W. Jiang, Epigenetic-mediated upregulation of progesterone receptor b gene in endometrial cancer cell lines, Gynecol Oncol 99 (1) (2005) 135-41. doi:10.1016/j.ygyno.2005.05.035.

[17] Y. Ren, X. Liu, D. Ma, Y. Feng, N. Zhong, Down-regulation of the progesterone receptor by the methylation of progesterone receptor gene in endometrial cancer cells, Cancer Genet Cytogenet 175 (2) (2007) 107-16. doi:10.1016/j.cancergencyto.2007.02.002.

[18] S. Yang, X. Xiao, Y. Jia, X. Liu, Y. Zhang, X. Wang, C. J. Winters, E. J. Devor, X. Meng, K. W. Thiel, K. K. Leslie, Epigenetic modification restores functional pr expression in endometrial cancer cells, Curr Pharm Des 20 (11) (2014) 1874-80.

[19] J. L. Hecht, G. L. Mutter, Molecular and pathologic aspects of endometrial carcinogenesis, J Clin Oncol 24 (29) (2006) 4783-91. doi:10.1200/JCO.2006.06.7173.

[20] H. Liang, L. W. T. Cheung, J. Li, Z. Ju, S. Yu, K. Stemke-Hale, T. Dogruluk, Y. Lu, X. Liu, C. Gu, W. Guo, S. E. Scherer, H. Carter, S. N. Westin, M. D. Dyer, R. G. W. Verhaak, F. Zhang, R. Karchin, C.-G. Liu, K. H. Lu, R. R. Broaddus, K. L. Scott, B. T. Hennessy, G. B. Mills, Wholeexome sequencing combined with functional genomics reveals novel candidate driver cancer genes in endometrial cancer, Genome Res 22 (11) (2012) 2120-9. doi:10.1101/gr.137596.112.

[21] A. Khan, X. Zhang, dbsuper: a database of super-enhancers in mouse and human genome, Nucleic Acids Res 44 (D1) (2016) D164-71. doi:10.1093/nar/gkv1002.

[22] D. Hnisz, J. Schuijers, C. Y. Lin, A. S. Weintraub, B. J. Abraham, T. I. Lee, J. E. Bradner, R. A. Young, Convergence of developmental and oncogenic signaling pathways at transcriptional super-enhancers, Mol Cell 58 (2) (2015) 362-70. doi:10.1016/j.molcel.2015.02.014.

[23] C. Ballaré, M. Uhrig, T. Bechtold, E. Sancho, M. Di Domenico, A. Migliaccio, F. Auricchio, M. Beato, Two domains of the progesterone receptor interact with the estrogen receptor and are required for progesterone activation of the c-src/erk pathway in mammalian cells, Mol Cell Biol 23 (6) (2003) 1994-2008.

[24] G. Vallejo, C. Ballaré, J. L. Barañao, M. Beato, P. Saragüeta, Progestin activation of nongenomic pathways via cross talk of progesterone receptor 
with estrogen receptor beta induces proliferation of endometrial stromal cells, Mol Endocrinol 19 (12) (2005) 3023-37. doi:10.1210/me.2005-0016.

[25] F. Le Dily, D. Baù, A. Pohl, G. P. Vicent, F. Serra, D. Soronellas, G. Castellano, R. H. G. Wright, C. Ballare, G. Filion, M. A. Marti-Renom, M. Beato, Distinct structural transitions of chromatin topological domains correlate with coordinated hormone-induced gene regulation, Genes Dev 28 (19) (2014) 2151-62. doi:10.1101/gad.241422.114.

[26] C. Ballaré, G. Castellano, L. Gaveglia, S. Althammer, J. González-Vallinas, E. Eyras, F. Le Dily, R. Zaurin, D. Soronellas, G. P. Vicent, M. Beato, Nucleosome-driven transcription factor binding and gene regulation, Mol Cell 49 (1) (2013) 67-79. doi:10.1016/j.molcel.2012.10.019.

[27] A. S. Nacht, A. Pohl, R. Zaurin, D. Soronellas, J. Quilez, P. Sharma, R. H. Wright, M. Beato, G. P. Vicent, Hormone-induced repression of genes requires brg1-mediated h1.2 deposition at target promoters, EMBO J 35 (16) (2016) 1822-43. doi:10.15252/embj.201593260.

[28] E. F. Need, L. A. Selth, A. P. Trotta, D. A. Leach, L. Giorgio, M. A. O'Loughlin, E. Smith, P. G. Gill, W. V. Ingman, J. D. Graham, G. Buchanan, The unique transcriptional response produced by concurrent estrogen and progesterone treatment in breast cancer cells results in upregulation of growth factor pathways and switching from a luminal a to a basal-like subtype, BMC Cancer 15 (2015) 791. doi:10.1186/s12885-015-1819-3.

[29] F. Le Dily, E. Vidal, Y. Cuartero, J. Quilez, A. S. Nacht, G. P. Vicent, J. Carbonell-Caballero, P. Sharma, J. L. Villanueva-Cañas, R. Ferrari, L. I. De Llobet, G. Verde, R. H. G. Wright, M. Beato, Hormone-control regions mediate steroid receptor-dependent genome organization, Genome Res 29 (1) (2019) 29-39. doi:10.1101/gr.243824.118.

[30] S. A. Lambert, A. Jolma, L. F. Campitelli, P. K. Das, Y. Yin, M. Albu, X. Chen, J. Taipale, T. R. Hughes, M. T. Weirauch, The human transcription factors, Cell 172 (4) (2018) 650-665. doi:10.1016/j.cell.2018.01.029.

[31] J. Gertz, T. E. Reddy, K. E. Varley, M. J. Garabedian, R. M. Myers, Genistein and bisphenol a exposure cause estrogen receptor 1 to bind thousands of sites in a cell type-specific manner, Genome Res 22 (11) (2012) 2153-62. doi:10.1101/gr.135681.111. 
[32] H. Hata, M. Hamano, A. Tsujii, Y. Togasaki, H. Kuramoto, M. Nishida, Y. Anzai, E. Gurpide, [in vitro study for hormones and growth factors dependent cell proliferation of endometrial adenocarcinoma cells], Hum Cell 6 (3) (1993) 182-7.

[33] M. Nishida, The ishikawa cells from birth to the present, Hum Cell 15 (3) (2002) 104-17.

[34] B. Mulac-Jericevic, R. A. Mullinax, F. J. DeMayo, J. P. Lydon, O. M. Conneely, Subgroup of reproductive functions of progesterone mediated by progesterone receptor-b isoform, Science 289 (5485) (2000) 1751-4. doi:10.1126/science.289.5485.1751.

[35] O. M. Conneely, B. Mulac-Jericevic, J. P. Lydon, Progesterone-dependent regulation of female reproductive activity by two distinct progesterone receptor isoforms, Steroids 68 (10-13) (2003) 771-8.

[36] P. A. Mote, R. L. Balleine, E. M. McGowan, C. L. Clarke, Colocalization of progesterone receptors a and b by dual immunofluorescent histochemistry in human endometrium during the menstrual cycle, J Clin Endocrinol Metab 84 (8) (1999) 2963-71. doi:10.1210/jcem.84.8.5928.

[37] H. Mohammed, I. A. Russell, R. Stark, O. M. Rueda, T. E. Hickey, G. A. Tarulli, A. A. Serandour, A. A. A. Serandour, S. N. Birrell, A. Bruna, A. Saadi, S. Menon, J. Hadfield, M. Pugh, G. V. Raj, G. D. Brown, C. D'Santos, J. L. L. Robinson, G. Silva, R. Launchbury, C. M. Perou, J. Stingl, C. Caldas, W. D. Tilley, J. S. Carroll, Progesterone receptor modulates er action in breast cancer, Nature 523 (7560) (2015) 313-7. doi:10.1038/nature14583.

[38] H. Singhal, M. E. Greene, G. Tarulli, A. L. Zarnke, R. J. Bourgo, M. Laine, Y.-F. Chang, S. Ma, A. G. Dembo, G. V. Raj, T. E. Hickey, W. D. Tilley, G. L. Greene, Genomic agonism and phenotypic antagonism between estrogen and progesterone receptors in breast cancer, Sci Adv 2 (6) (2016) e1501924. doi:10.1126/sciadv.1501924.

[39] P. A. Sanderson, H. O. D. Critchley, A. R. W. Williams, M. J. Arends, P. T. K. Saunders, New concepts for an old problem: the diagnosis of endometrial hyperplasia, Hum Reprod Update 23 (2) (2017) 232-254. doi:10.1093/humupd/dmw042. 
[40] C. M. Quick, A. R. Laury, N. M. Monte, G. L. Mutter, Utility of pax2 as a marker for diagnosis of endometrial intraepithelial neoplasia, Am J Clin Pathol 138 (5) (2012) 678-84. doi:10.1309/AJCP8OMLT7KDWLMF.

[41] E. Cannavò, P. Khoueiry, D. A. Garfield, P. Geeleher, T. Zichner, E. H. Gustafson, L. Ciglar, J. O. Korbel, E. E. M. Furlong, Shadow enhancers are pervasive features of developmental regulatory networks, Curr Biol 26 (1) (2016) 38-51. doi:10.1016/j.cub.2015.11.034.

[42] X. Zhang, P. S. Choi, J. M. Francis, M. Imielinski, H. Watanabe, A. D. Cherniack, M. Meyerson, Identification of focally amplified lineage-specific super-enhancers in human epithelial cancers, Nat Genet 48 (2) (2016) 17682. doi:10.1038/ng.3470.

[43] P. Chomczynski, N. Sacchi, Single-step method of rna isolation by acid guanidinium thiocyanate-phenol-chloroform extraction, Anal Biochem 162 (1) (1987) 156-9. doi:10.1006/abio.1987.9999.

[44] C. Trapnell, L. Pachter, S. L. Salzberg, Tophat: discovering splice junctions with rna-seq, Bioinformatics 25 (9) (2009) 1105-11. doi:10.1093/bioinformatics/btp120.

[45] C. Trapnell, B. A. Williams, G. Pertea, A. Mortazavi, G. Kwan, M. J. van Baren, S. L. Salzberg, B. J. Wold, L. Pachter, Transcript assembly and quantification by rna-seq reveals unannotated transcripts and isoform switching during cell differentiation, Nat Biotechnol 28 (5) (2010) 511-5. doi:10.1038/nbt.1621.

[46] C. Fresno, E. A. Fernández, Rdavidwebservice: a versatile r interface to david, Bioinformatics 29 (21) (2013) 2810-1. doi:10.1093/bioinformatics/btt487.

[47] H. Strutt, R. Paro, Mapping dna target sites of chromatin proteins in vivo by formaldehyde crosslinking, Methods Mol Biol 119 (1999) 455-67. doi:10.1385/1-59259-681-9:455.

[48] G. P. Vicent, A. S. Nacht, J. Font-Mateu, G. Castellano, L. Gaveglia, C. Ballaré, M. Beato, Four enzymes cooperate to displace histone h1 during the first minute of hormonal gene activation, Genes Dev 25 (8) (2011) 845-62. doi:10.1101/gad.621811.

[49] A. M. Bolger, M. Lohse, B. Usadel, Trimmomatic: a flexible trimmer for illumina sequence data, Bioinformatics 30 (15) (2014) 2114-20. doi:10.1093/bioinformatics/btu170. 
[50] H. Li, R. Durbin, Fast and accurate short read alignment with burrows-wheeler transform, Bioinformatics 25 (14) (2009) 1754-60. doi:10.1093/bioinformatics/btp324.

[51] Y. Zhang, T. Liu, C. A. Meyer, J. Eeckhoute, D. S. Johnson, B. E. Bernstein, C. Nusbaum, R. M. Myers, M. Brown, W. Li, X. S. Liu, Model-based analysis of chip-seq (macs), Genome Biol 9 (9) (2008) R137. doi:10.1186/gb-2008-9-9r137.

[52] A. R. Quinlan, Bedtools: The swiss-army tool for genome feature analysis, Curr Protoc Bioinformatics 47 (2014) 11.12.1-34. doi:10.1002/0471250953.bi1112s47.

[53] I. Kremsky, N. Bellora, E. Eyras, A quantitative profiling tool for diverse genomic data types reveals potential associations between chromatin and pre-mrna processing, PLoS One 10 (7) (2015) e0132448. doi:10.1371/journal.pone.0132448.

[54] T. L. Bailey, J. Johnson, C. E. Grant, W. S. Noble, The meme suite, Nucleic Acids Res 43 (W1) (2015) W39-49. doi:10.1093/nar/gkv416.

[55] E. Agirre, N. Bellora, M. Alló, A. Pagès, P. Bertucci, A. R. Kornblihtt, E. Eyras, A chromatin code for alternative splicing involving a putative association between ctcf and hp1 proteins, BMC Biol 13 (2015) 31. doi:10.1186/s12915-015-0141-5.

[56] M. T. Weirauch, A. Yang, M. Albu, A. G. Cote, A. MontenegroMontero, P. Drewe, H. S. Najafabadi, S. A. Lambert, I. Mann, K. Cook, H. Zheng, A. Goity, H. van Bakel, J.-C. Lozano, M. Galli, M. G. Lewsey, E. Huang, T. Mukherjee, X. Chen, J. S. Reece-Hoyes, S. Govindarajan, G. Shaulsky, A. J. M. Walhout, F.-Y. Bouget, G. Ratsch, L. F. Larrondo, J. R. Ecker, T. R. Hughes, Determination and inference of eukaryotic transcription factor sequence specificity, Cell 158 (6) (2014) 1431-1443. doi:10.1016/j.cell.2014.08.009.

[57] J. D. Buenrostro, P. G. Giresi, L. C. Zaba, H. Y. Chang, W. J. Greenleaf, Transposition of native chromatin for fast and sensitive epigenomic profiling of open chromatin, dna-binding proteins and nucleosome position, Nat Methods 10 (12) (2013) 1213-8. doi:10.1038/nmeth.2688.

[58] E. Lieberman-Aiden, N. L. van Berkum, L. Williams, M. Imakaev, T. Ragoczy, A. Telling, I. Amit, B. R. Lajoie, P. J. Sabo, M. O. Dorschner, 
R. Sandstrom, B. Bernstein, M. A. Bender, M. Groudine, A. Gnirke, J. Stamatoyannopoulos, L. A. Mirny, E. S. Lander, J. Dekker, Comprehensive mapping of long-range interactions reveals folding principles of the human genome, Science 326 (5950) (2009) 289-93. doi:10.1126/science.1181369.

\section{Data Availability}

Sequencing data will be available for download from GEO shortly.

\section{Acknowledgments}

We are grateful to members of the Beato and Saragüeta laboratories for help and suggestions.

\section{Author Contributions}

ALG, FLD, MB and PS design experiments. ALG, RJ, GV and ITR performed cell culture and experiments. ALG, RJ, NB, GM, CF, JQO and FLD performed bioinformatic analyses. RJ, JQO and FLD analyzed HiC results. GV, EF, GV, MB, ALG and PS discussed experiments and manuscript. MB and PS provided fundings for this paper. ALG, MB and PS wrote the manuscript.

\section{Funding}

This work was supported by the National Scientific and Technical Research Council (CONICET), Grant/Award Number: PIP 2015-682; Scientific and Technical Research Fund (FONCyT), Grant/Award Number: PICT 2015-3426. ERC-MINCYT international cooperation program. Doctoral Fellowship from CONICET, Argentina, awarded to ALG. PS is PI from CONICET.

\section{Competing interest}

None declared. 
A

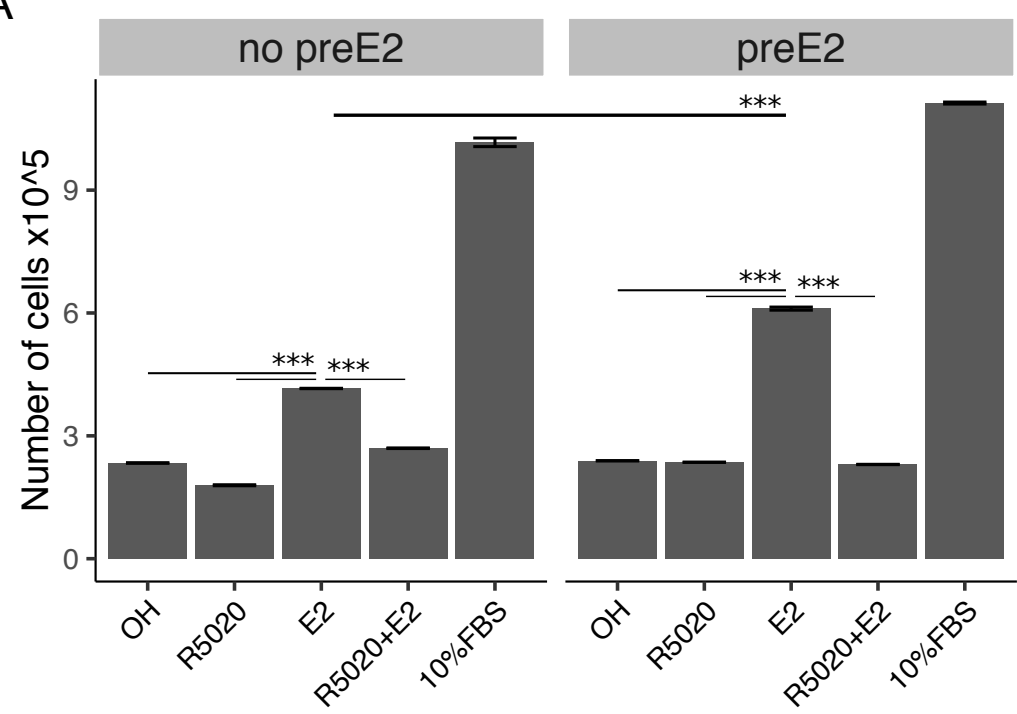

B

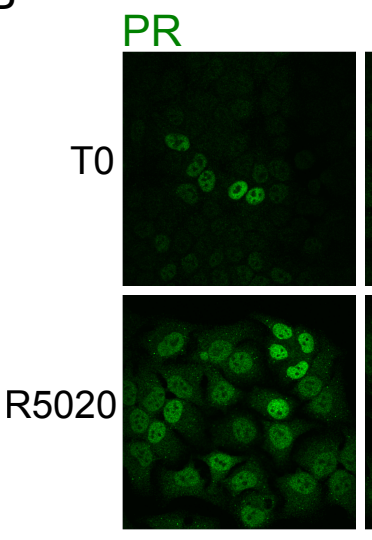

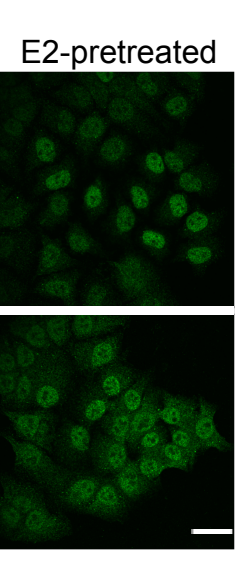

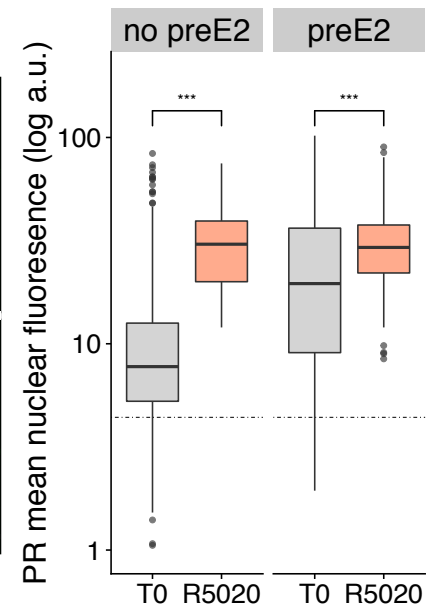

C

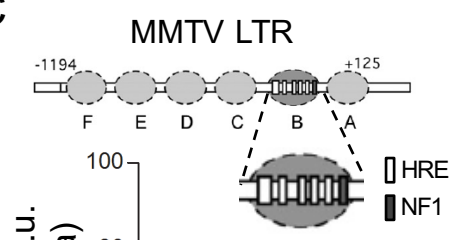

D
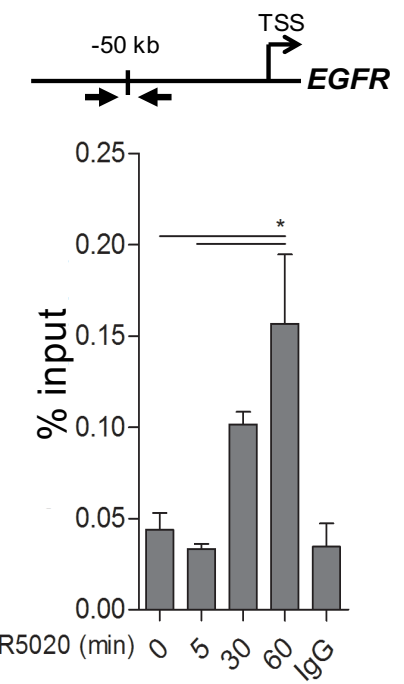

Figure 1. R5020 inhibits E2-induced Ishikawa cell proliferation through an active PR that is capable of transactivating an exogenous MMTV promoter sequence and an endogenous enhancer sequence located 50kb upstream of EGFR gene. (A) Proliferation of Ishikawa cells either pretreated with E2 10nM for 12h (preE2) or not (no preE2) and later treated with vehicle (OH), E2 10nM (E2), R5020 10nM (R5020), E2 combined with R5020 $(\mathrm{E} 2+\mathrm{R} 5020)$ and FBS $(10 \% \mathrm{FBS})$, expressed as mean number of cells $\pm \mathrm{SE}$ of three independent experiments. $(* * *) \mathrm{p}<0.001$. (B) Immunofluorescence of PR in untreated (T0; top left), 60min R5020-treated (R5020; bottom left), 12h E2-pretreated (top right) and 12h E2-pretreated 60min R5020-treated (bottom right) Ishikawa cells. Scale bar is equivalent to $30 \mu \mathrm{m}$. Mean nuclear signal of PR for every cell in all images was determined and shown to the right of the images as arbitrary units (log a.u.). Horizontal dashed lines in boxplots indicate background signal for secondary antibody. (***) p<0.001. (C) Ishikawa cells transfected with an MMTV-Luciferase reporter gene and treated with vehicle (OH) and R5020 10nM (R5020) for 18h Diagram at the top depicts MMTV LTR promoter features, including several hormone response elements (HRE) and a nuclear factor 1 (NF1) binding site within nucleosome B (dark grey circle and magnification). Numbers in the diagram indicate base pair position relative to transcription start site (TSS). Results are expressed as relative units (r.u.) of Luciferase activity. (D) Representation of EGFR TSS and the enhancer sequence located 50kb upstream used to evaluate PR recruitment. Black arrows indicate position of qPCR primers employed on samples treated or not (0) with R5020 for 5, 30 and 60min. Unspecific immunoprecipitation of chromatin was performed in parallel with normal rabbit IgG (IgG). Results are expressed as \%input DNA and bars represent mean fold change in PR enrichment relative to time 0 (untreated cells) \pm SE of two independent experiments. $(*) \mathrm{p}<0.05$. 
A

\begin{tabular}{|c|c|}
\hline R5020 (min) & PR \\
\hline 0 & 331 \\
\hline 5 & 275 \\
\hline 30 & 1446 \\
\hline 60 & 1491 \\
\hline \multicolumn{2}{|c|}{ Number of ERbs } \\
\hline E2 (min) & ER \\
\hline 0 & 25 \\
\hline 5 & 178 \\
\hline 60 & 1591 \\
\hline 360 & 1973 \\
\hline
\end{tabular}

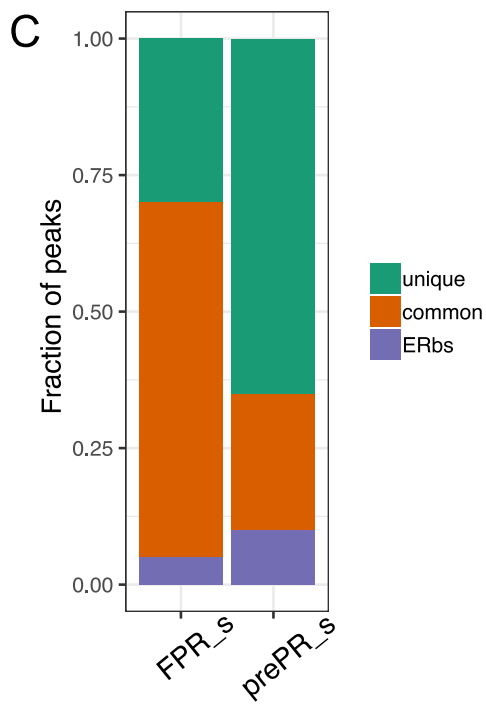

Number of PRbs

\begin{tabular}{cc} 
prePR & FPR \\
\hline 346 & 476 \\
- & 499 \\
2745 & 3913 \\
5701 & 3128 \\
\hline
\end{tabular}

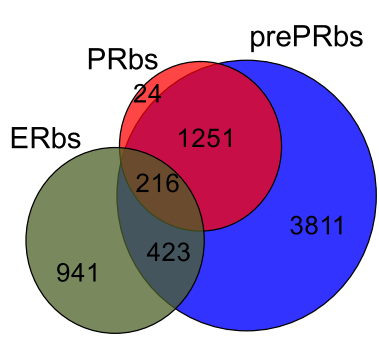

D

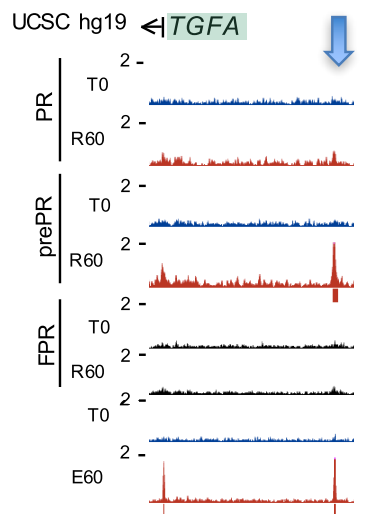

B
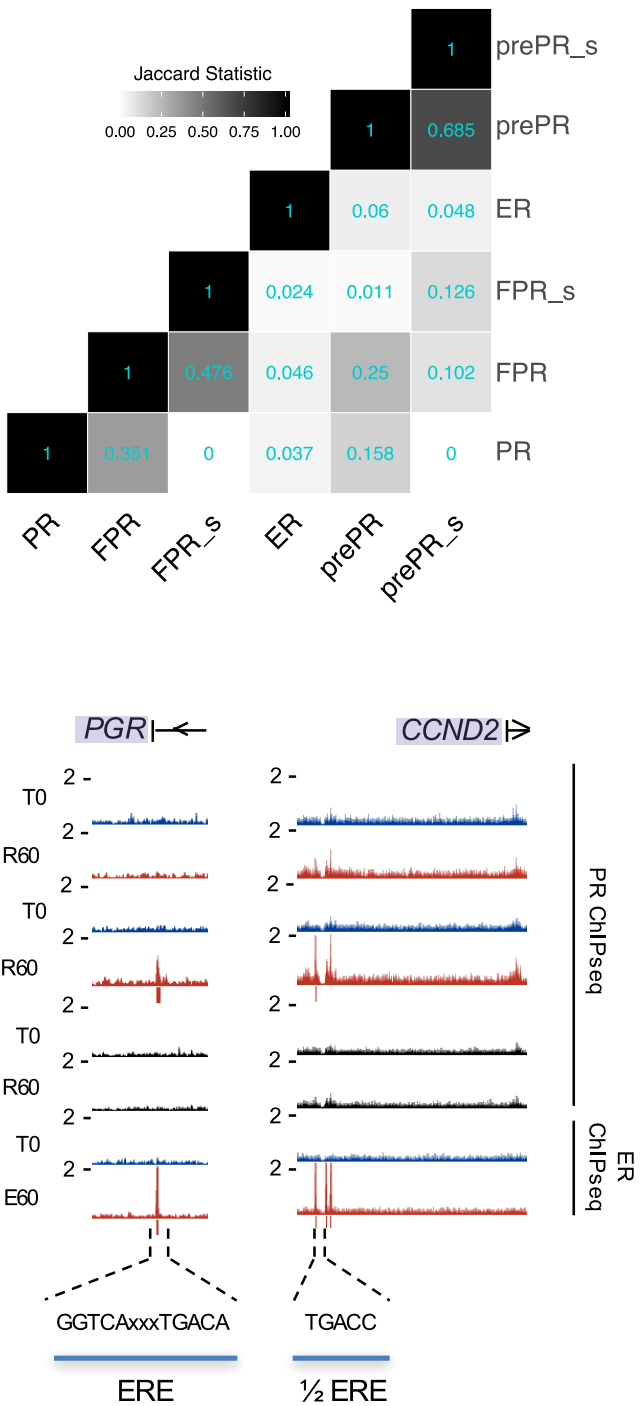

Figure 2. Estradiol induces R5020-dependent PR binding to specific regions in chromatin. (A) Upper table shows total number of PRbs obtained by ChIPseq for untreated (0min) and R5020-treated (5,30 and 60min) endometrial Ishikawa cells under three different conditions: non-pretreated with E2 (PR), pretreated with E2 for 12h (prePR) and exogenous expression of PR (FPR). Lower table shows number of ERbs using anti-ER $\alpha$ antibody on untreated (0min) and E2-treated (5, 60 and 360min) Ishikawa cells. Venn Diagram shows shared binding sites among PRbs (red), prePRbs (blue) and ERbs (green). (B) Similarity matrix built by calculating Jaccard statistic over all samples from A and FPR_s and prePR_s, in which PRbs were subtracted. Jaccard value of 1 indicates maximum similarity. (C) Fraction of peaks in FPR_s and prePR_s that are not shared with each other (unique), that are common to each other (common) and that are common with ER (ERbs). (D) Graphic visualization of PR and ER binding in untreated (T0) and 60min hormone-treated (R60 and E60) Ishikawa cells. Peak height equals normalized number of reads and lines below the peaks indicate statistical significance determined by MACS. Black horizontal arrows symbolize the beginning and orientation of the genes and blue vertical line points to the peak of interest. R60: 60min R5020 10nM; E60: 60min E2 10nM. The three regions displayed include TGFA, PGR and CCND2 genes (indicated at the top). An estrogen response element (ERE) and a half ERE are indicated below the peaks. 


\section{La_Greca_Fig3}

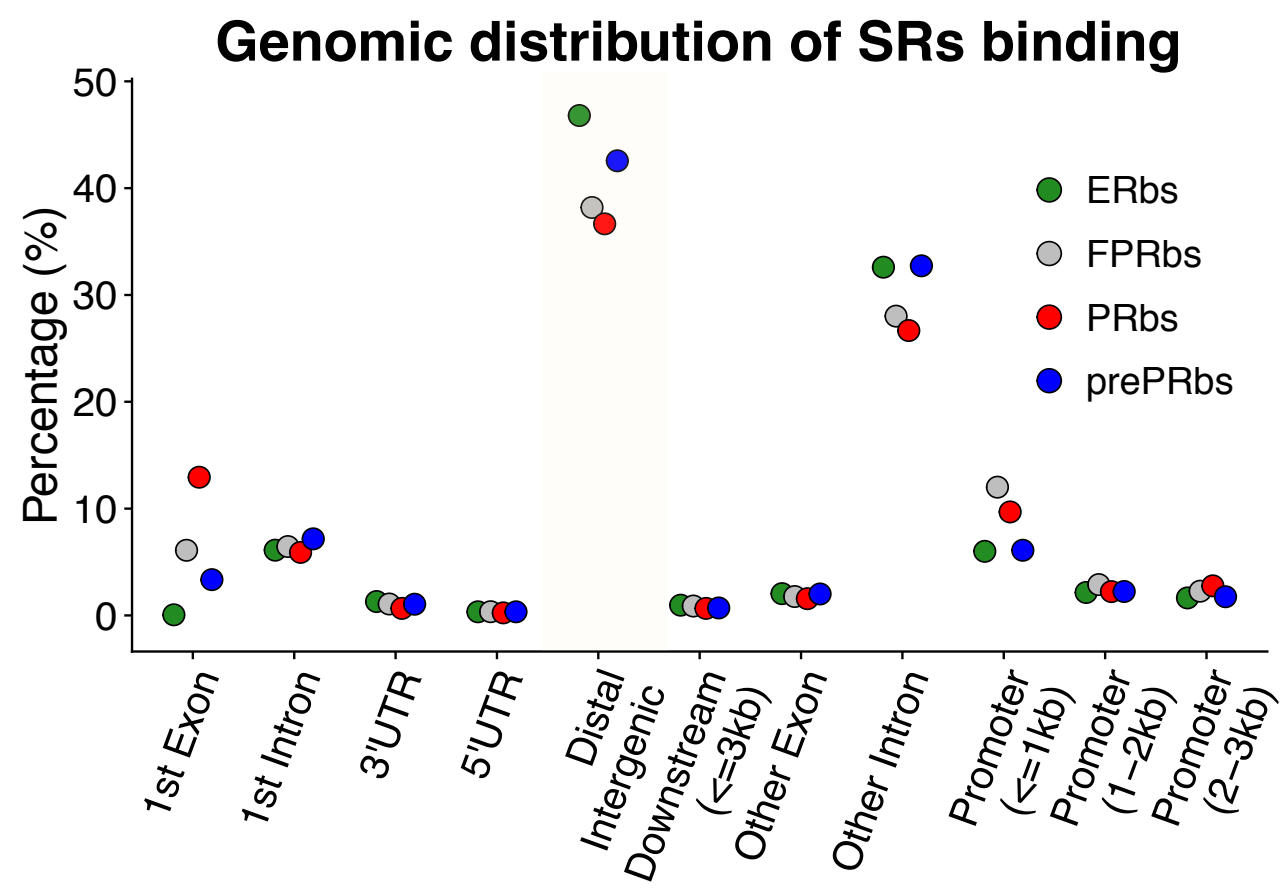

\begin{tabular}{|c|c|c|c|c|c|c|c|c|c|c|c|}
\hline PRbs & 12.9 & 5.9 & 0.7 & 0.2 & 36.7 & 0.7 & 1.6 & 26.7 & 9.7 & 2.2 & 2.8 \\
\hline FPRbs & 6.1 & 6.5 & 1.1 & 0.4 & 38.2 & 0.9 & 1.8 & 28 & 12 & 2.9 & 2.3 \\
\hline ERbs & 0.1 & 6.1 & 1.3 & 0.3 & 46.8 & 1 & 2 & 32.6 & 6 & 2.2 & 1.6 \\
\hline ePRbs & 3.4 & 7.2 & 1.1 & 0.3 & 42.6 & 0.7 & 2 & 32.7 & 6.1 & 2.2 & 1.8 \\
\hline
\end{tabular}

Figure 3. PR and ER $\alpha$ mediate hormone-dependent gene expression through binding regions other than promoters. Classification of steroid receptor binding relative to genomic features expressed as percentage (\%) of peaks after $60 \mathrm{~min}$ of hormone treatment inside each feature. Legend at the top right corner indicates the color key for ERbs (green dots) and three conditions of PR binding: non-pretreated with E2 (PRbs, red dots), pretreated with E2 for 12h (prePRbs, blue dots) and exogenous expression of PR (FPRbs, grey dots). The table below shows percentages represented in the plot. 
A

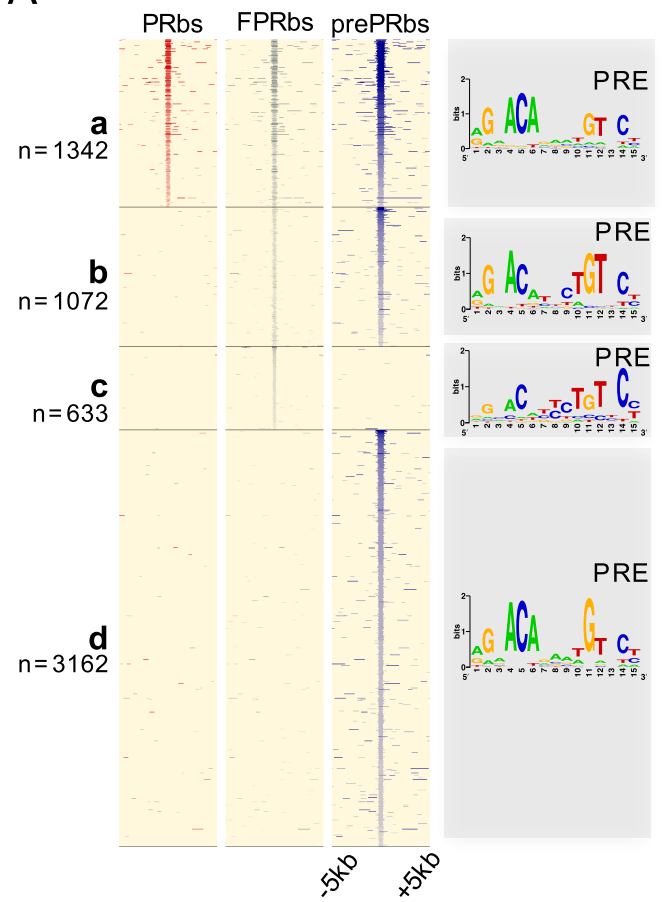

B

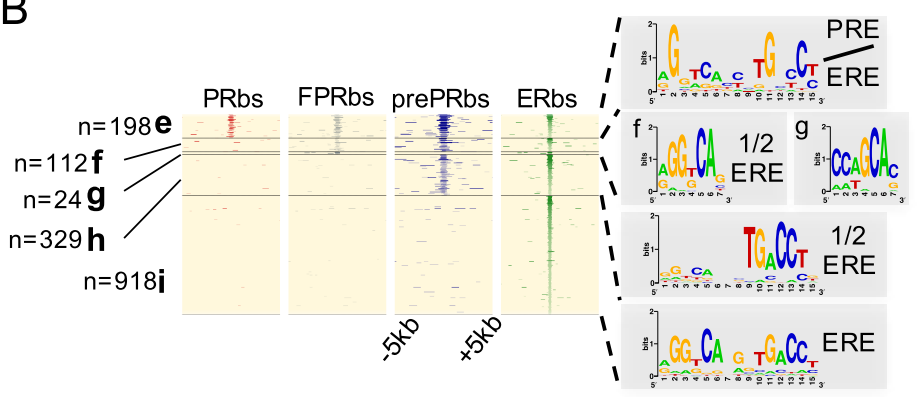

C

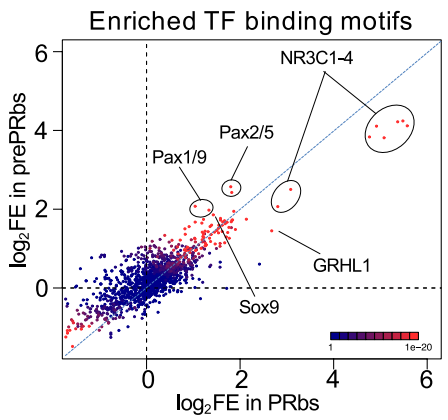

D

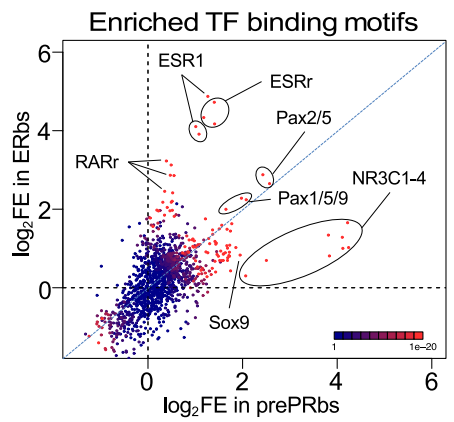

Figure 4. A fraction of E2-induced PRbs localize on ERbs and contain half ERE motifs. (A) Peak signals in PRbs, FPRbs and prePRbs from 60min R5020-treated Ishikawa cells were plotted as heatmaps. Regions were defined inside a window of $10 \mathrm{~kb}$ centered in peak summit $( \pm 5 \mathrm{~kb})$ and intensity of the signal correspond to number of reads in each region. Heatmap is subdivided into 4 mutually exclusive groups depending on shared/partly shared/non-shared binding sites: a ( $\mathrm{n}=$ $1342)$, sites shared by all three conditions of PR binding; $b(n=1072)$, sites uniquely found in FPR and prePR; $c(n=633)$, sites found only in FPR; and d $(n=3162)$, sites found only in prePR. De novo motif discovery (MEME) was performed on all groups and results are indicated as sequence logos to the right of the map, including the name of the most related known motif. PRE: progesterone response element. (B) Peak signals in PRbs, FPRbs and prePRbs as in A, and ERbs from 60min E2-treated Ishikawa cells. Heatmap was subdivided into 5 mutually exclusive groups: e $(n=198)$, sites shared by all three conditions of PR binding and ER binding; $\mathrm{f}(\mathrm{n}=112)$, sites shared by FPRbs, prePRbs and ERbs; $\mathrm{g}(\mathrm{n}=24)$, sites shared by FPRbs and ERbs; $h(n=329)$, sites shared by prePRbs and ERbs; and i $(n=918)$, sites uniquely found in ERbs. Motif discovery was performed as in A for all groups and results are shown to the right of the map, including the most related known motif. ERE: estrogen response element; $\frac{1}{2}$ ERE: half ERE. (C) Fold enrichment values $(\log 2 \mathrm{FE})$ of 1.395 known TF binding motifs on prePRbs and PRbs. Combined p-values for enrichment analyses are indicated through the color key displayed at the lower right corner of the plot. Relevant motifs pointed on the plot correspond to NR3C1-4, members of the PAX family (1, 2, 5 and 9) and SOX9. (D) Comparison as in C between prePRbs and ERbs. Relevant motifs pointed on the plot correspond to NR3C1-4, members of the PAX family (1, 2, 5 and 9), SOX9, ESR1 and estrogen related (ESRr) and retinoic acid receptor $(\mathrm{RARr})$. 
bioRxiv preprint doi: https://doi.org/10.1101/739466; this version posted August 20, 2019. The copyright holder for this preprint (which was not certified by peer review) is the author/funder. All rights reserved. No reuse allowed without permission.

\section{La_Greca_Fig5}

A
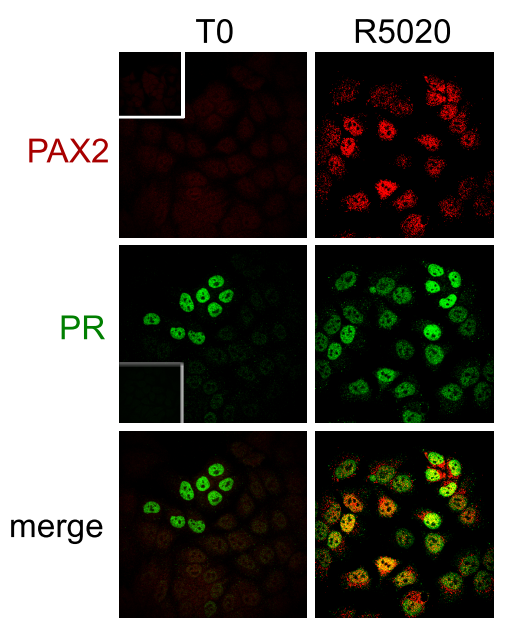

\section{B}

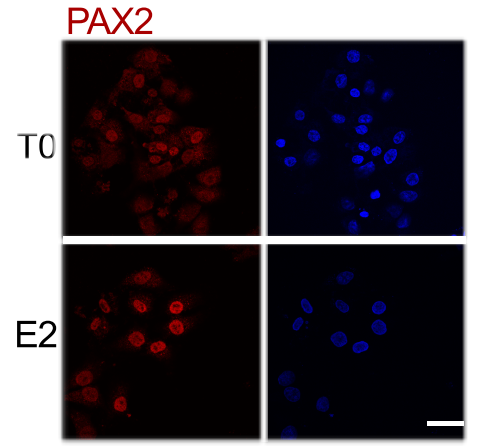

E2-pretreated
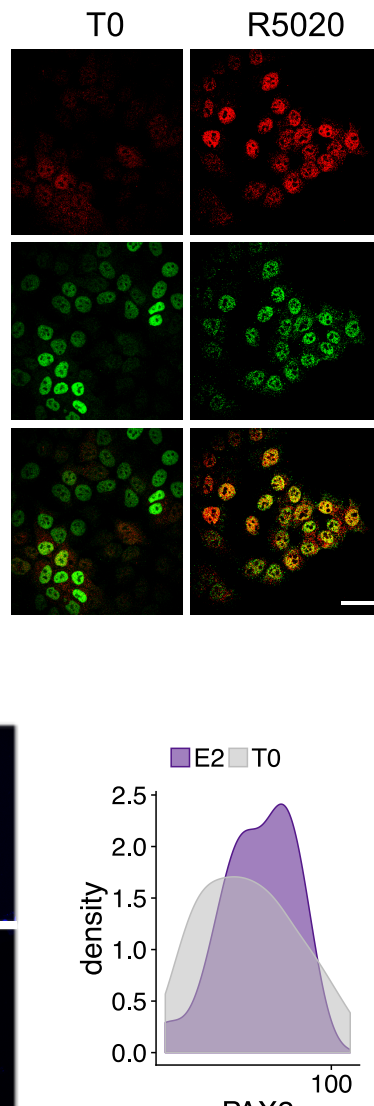

PAX2
C

PAX2 binding sites: R502060min

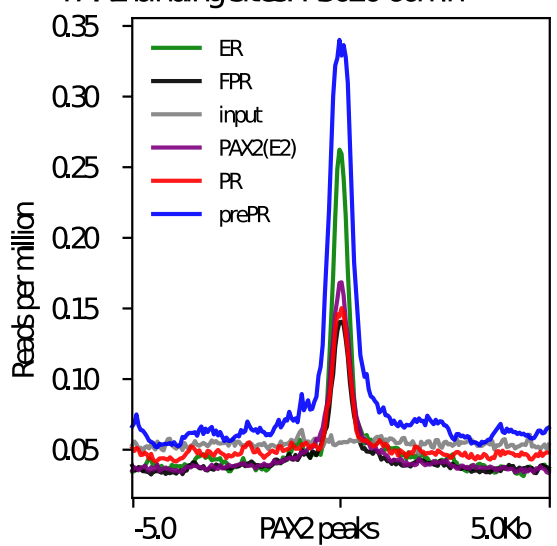

D PAX2 binding sites: E260min

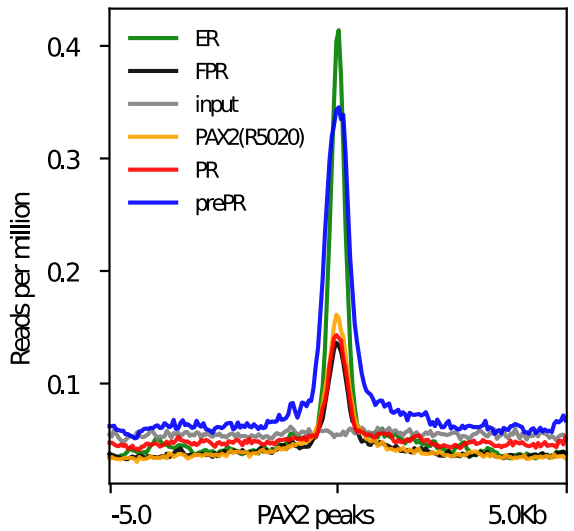

Figure 5. PAX2 co-localizes with PR and ER $\alpha$ in nuclei of Ishikawa cells and it is positioned primarily in the vicinity of receptors binding sites. (A) Immunofluorescent detection of PR (green) and PAX2 (red) in untreated (T0) and 60min R5020-treated (R5020) Ishikawa cells which were pretreated or not with E2 for 12h (non-pretreated, E2-pretreated) Images were merged for co-localization analysis (merge). Scale bar is shown in the panels and is equivalent to $30 \mu \mathrm{m}$. (B) Representative images of PAX2 signal (red) in cells treated or not with E2 for 60min and dapi staining (blue). Scale bar is shown in the figure and equals to $30 \mu \mathrm{m}$. Distribution of intensities in nuclear signal for both conditions is shown to the right of the images. (C) Binding profiles of ER (green), PR (red), FPR (black) and prePR (blue) on PAX2 binding sites of 60min R5020-treated Ishikawa cells. PAX2 binding after 60min E2 treatment was included (purple) (D) Binding profiles as in (C) on PAX2 binding sites of 60min E2-treated Ishikawa cells. PAX2 binding after 60min R5020 treatment was included (orange). 
A

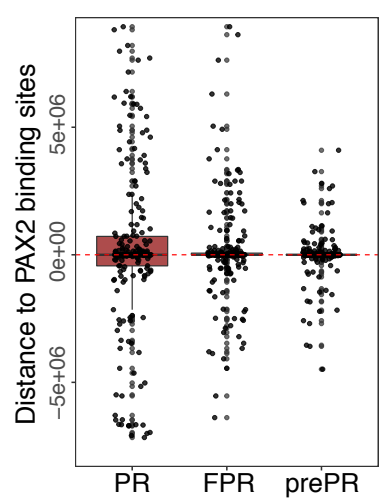

B

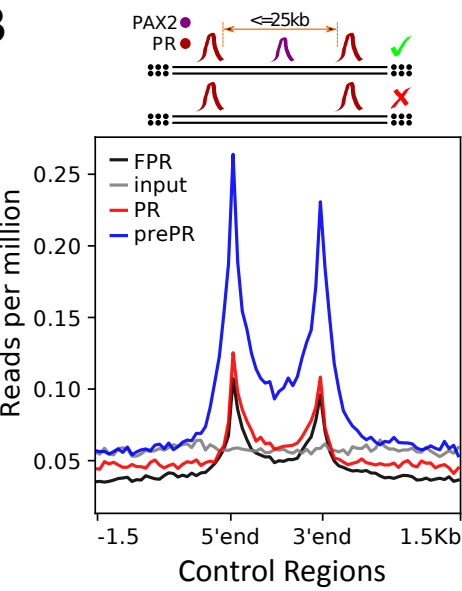

E

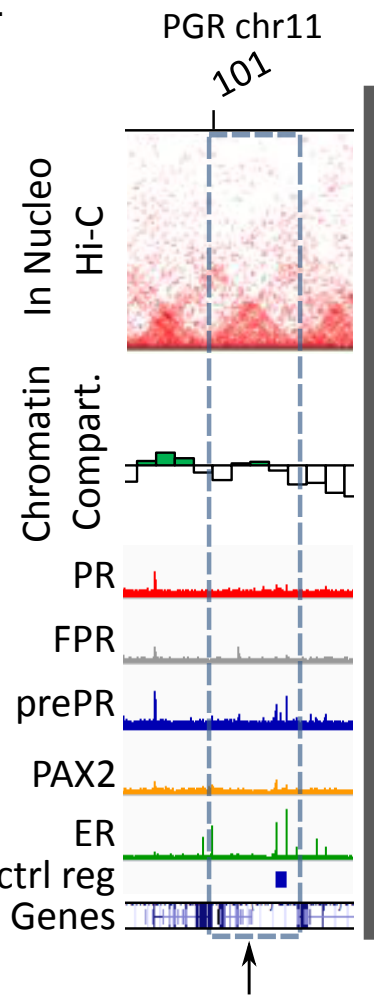

control regions
C

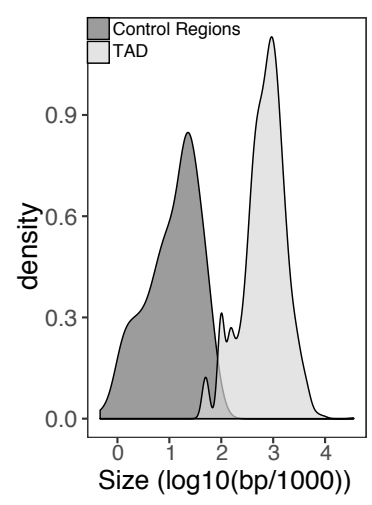

$\mathrm{D}$

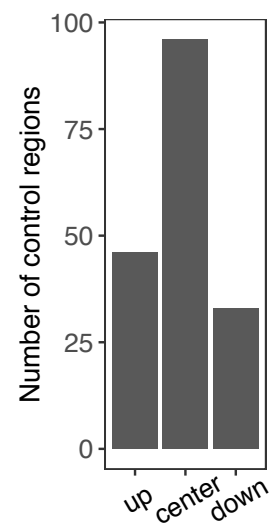

$\mathrm{F}$ ATAC-seq
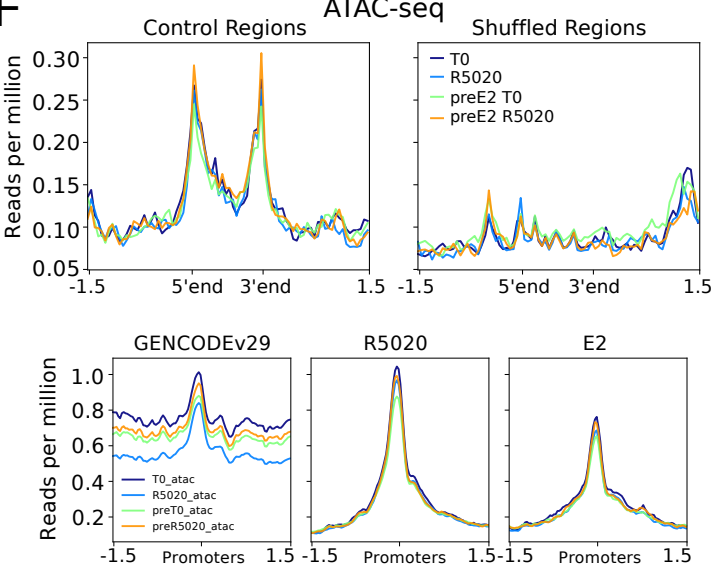

G

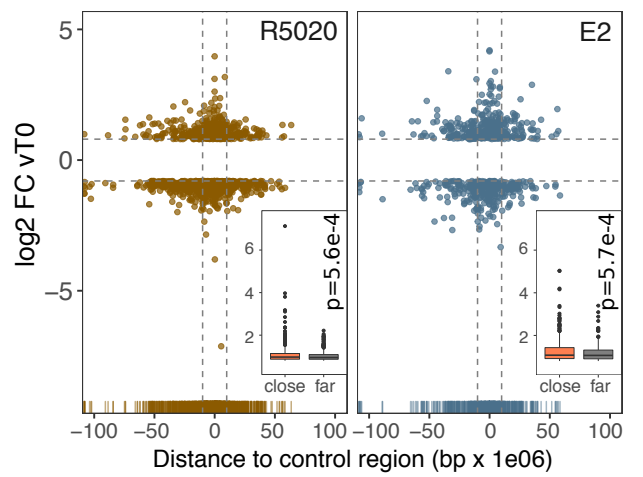

Figure 6. Convergence of PR and Pax2 binding in TADs with regulated genes defines potential endometrial regulatory domains. (A) Relative distance from PRbs, FPRbs and prePRbs to PAXbs (both E2 and R5020 originated sites). Red dashed line marks overlapping events (distance $=0)$. Peaks located upstream of PAXbs are noted as negative distance values, while downstream peaks are positive. (B) Coverage profiles of PR (red), FPR (black) and prePR (blue) binding on control regions delimited by the start and end labels. Input sample (grey) was included in the plot. Rules for qualifying as a control region are depicted on top of the profile plot. (C) Size distribution of Control Regions and TADs, expressed as $\log 10$. (D) Distribution of control regions in harbouring TADs divided into center (CENTER), upstream end (UP) and downstream end (DOWN). (E) Upper panel shows the contact matrices at a resolution of 20kb obtained by In Nucleo Hi-C in PGR and ALPP loci. Middle panel shows the spatial segregation of chromatin as open or closed compartments inside TADs (green bars: A compartment; white bars: B compartment - see methods section). The bottom panels show ChIPseq signal distribution of PR, FPR, prePR, PAX2 and ER $\alpha$ as well as the location of Control Regions (ctrl reg) and genes over the region. The dashed rectangle restricts the TAD of interest and the vertical arrow marks the TSS of $P G R$ and ALPP. Magnified images over Control Regions are shown to the right. (F) ATAC-seq peaks from cells untreated (T0), treated with R5020 for 60min, 12h E2-pretreated (preE2 T0) and E2-pretreated followed by 60min treatment with R5020. Signal was ploted over Control Regions, shuffled Control Regions (Shuffled Regions), promoters of all annotated genes from GENCODE database (GENCODEv29) and promoters of genes regulated by 12h treatments with R5020 or E2. (G) Plot shows fold change values of genes regulated by R5020 and E2 (v. untreated cells) relative to Control Regions. Genes located upstream of Control Regions are represented with negative distance values. Dashed horizontal lines mark fold change cut-off points $(\log 2 \mathrm{FC}= \pm 0.8)$ and vertical lines are placed at position -10 and $10 \mathrm{Mb}$. Insets depict comparison of fold change values (absolute values) between genes located beneath (close) and over (far) a 10Mb distance from Control Regions. Statistical significance for this comparison was determined with Welch Two Sample t-test and is represented by a p value on the plot. 